\title{
Mechanical Proof of the Maxwell-Boltzmann Speed Distribution With Analytical Integration
}

\author{
Hejie $\operatorname{Lin}^{1} \&$ Tsung-Wu Lin $^{2}$ \\ ${ }^{1}$ Department of Mechanical Engineering, Oakland University, Rochester Hills, Michigan, USA \\ ${ }^{2}$ Department of Civil Engineering, National Taiwan University, Taipei, Taiwan \\ Correspondence: Hejie Lin, Department of Mechanical Engineering, Oakland University, Rochester Hills, Michigan, \\ USA. E-mail: hejielin@oakland.edu
}

Received: March 4, 2021 Accepted: April 22, $2021 \quad$ Online Published: April 24, 2021

doi:10.5539/ijsp.v10n3p135 URL: https://doi.org/10.5539/ijsp.v10n3p135

\begin{abstract}
The Maxwell-Boltzmann speed distribution is the probability distribution that describes the speeds of the particles of ideal gases. The Maxwell-Boltzmann speed distribution is valid for both un-mixed particles (one type of particle) and mixed particles (two types of particles). For mixed particles, both types of particles follow the Maxwell-Boltzmann speed distribution. Also, the most probable speed is inversely proportional to the square root of the mass.

The Maxwell-Boltzmann speed distribution of mixed particles is based on kinetic theory; however, it has never been derived from a mechanical point of view. This paper proves the Maxwell-Boltzmann speed distribution and the speed ratio of mixed particles based on probability analysis and Newton's law of motion. This paper requires the probability density function (PDF) $\psi^{a b}\left(u_{a} ; v_{a}, v_{b}\right)$ of the speed $u_{a}$ of the particle with mass $M_{a}$ after the collision of two particles with mass $M_{a}$ in speed $v_{a}$ and mass $M_{b}$ in speed $v_{b}$. The PDF $\psi^{a b}\left(u_{a} ; v_{a}, v_{b}\right)$ in integral form has been obtained before. This paper further performs the exact integration from the integral form to obtain the PDF $\psi^{a b}\left(u_{a} ; v_{a}, v_{b}\right)$ in an evaluated form, which is used in the following equation to get new distribution $\mathrm{P}_{\text {new }}^{a}\left(u_{a}\right)$ from old distributions $\mathrm{P}_{\text {old }}^{a}\left(v_{a}\right)$ and $\mathrm{P}_{\text {old }}^{b}\left(v_{b}\right)$. When $\mathrm{P}_{\text {old }}^{a}\left(v_{a}\right)$ and $\mathrm{P}_{\text {old }}^{b}\left(v_{b}\right)$ are the Maxwell-Boltzmann speed distributions, the integration $\mathrm{P}_{n e w}^{a}\left(u_{a}\right)$ obtained analytically is exactly the Maxwell-Boltzmann speed distribution.

$$
\mathrm{P}_{\text {new }}^{a}\left(u_{a}\right)=\int_{0}^{\infty} \int_{0}^{\infty} \psi^{a b}\left(u_{a} ; v_{a}, v_{b}\right) \mathrm{P}_{\text {old }}^{a}\left(v_{a}\right) \mathrm{P}_{\text {old }}^{b}\left(v_{b}\right) \mathrm{d} v_{a} \mathrm{~d} v_{b}, \quad a, b=1 \text { or } 2
$$

The mechanical proof of the Maxwell-Boltzmann speed distribution presented in this paper reveals the unsolved mechanical mystery of the Maxwell-Boltzmann speed distribution since it was proposed by Maxwell in 1860. Also, since the validation is carried out in an analytical approach, it proves that there is no theoretical limitation of mass ratio to the Maxwell-Boltzmann speed distribution. This provides a foundation and methodology for analyzing the interaction between particles with an extreme mass ratio, such as gases and neutrinos.
\end{abstract}

Keywords: Maxwell speed distribution, Maxwell-Boltzmann speed distribution, Maxwell-Boltzmann distribution, Avogadro's law, kinetic theory of gases, kinetic theory, thermodynamics, statistical mechanics, subatomic particles

\section{Overview}

James C. Maxwell (1860a,b) first provided the Maxwell speed distribution in 1860 on a statistical heuristic basis. Maxwell (1867) and Boltzmann (1872) carried out more investigations into the physical meaning of the distribution. Boltzmann (1877) derived the distribution again based on statistical thermodynamics. Nevertheless, none of their approaches were based on Newton's law of motion.

The simplest way to prove the Maxwell-Boltzmann speed distribution is from a statistical view, beginning from the normal distribution of the velocity $v_{x}$ in x-direction as follows.

$$
P\left(v_{x}\right)=\frac{1}{\sigma \sqrt{2 \pi}} e^{\frac{-1}{2}\left(\frac{v_{x}}{\sigma}\right)^{2}}
$$

or

$$
P\left(v_{x}\right)=\frac{h}{\sqrt{\pi}} e^{-h^{2} v_{x}^{2}}
$$


Where $\sigma=\frac{1}{\sqrt{2} h}, h=\sqrt{\frac{M}{2 k T}}, k$ is the Boltzmann constant, $T$ is the equilibrium temperature, and $M$ is the particle mass. Extending from the velocity $v_{x}$ to three independent velocities $\left(v_{x}, v_{y}, v_{z}\right)$ in three directions, and transferring it to spherical coordinates $(v, \theta, \varphi)$ using $v_{x}=v \sin \theta \cos \varphi, v_{y}=v \sin \theta \sin \varphi$, and $v_{z}=v \cos \theta$ gives

$$
\begin{aligned}
& \int_{-\infty}^{\infty} \int_{-\infty}^{\infty} \int_{-\infty}^{\infty} P\left(v_{x}\right) P\left(v_{y}\right) P\left(v_{z}\right) d v_{x} d v_{y} d v_{z} \\
& =\int_{0}^{\infty} \frac{4 h^{3}}{\sqrt{\pi}} v^{2} e^{-h^{2} v^{2}} d v=\int_{0}^{\infty} P(v) d v
\end{aligned}
$$

Where $P(v)$ is the Maxwell-Boltzmann speed distribution shown in Equation (4) (Brush, 1966, Landau et al., 1969, McQuarrie, 1976, Garrod, 1995, Maudlin, 2013).

$$
P(v)=\frac{4 h^{3}}{\sqrt{\pi}} v^{2} e^{-h^{2} v^{2}}
$$

In the Maxwell-Boltzmann speed distribution, the most probable speed, $\boldsymbol{v}_{\boldsymbol{m} \boldsymbol{p}}$, is inversely proportional to the square root of the mass for fixed temperatures as follows

$$
v_{m p}=\frac{1}{h}=\sqrt{\frac{2 k T}{M}}
$$

Therefore, when two types of particles with mass $M_{1}$ and $M_{2}$ are mixed at the same temperature, the above equation gives the following mass-speed relationship

$$
\frac{v_{1, m p}}{v_{2, m p}}=\sqrt{\frac{M_{2}}{M_{1}}}
$$

An example of the theoretical Maxwell-Boltzmann speed distribution curves and their corresponding most probable speeds $v_{1, m p}$ and $v_{2, m p}$ of two types of particles with a mass ratio of nine are shown below.

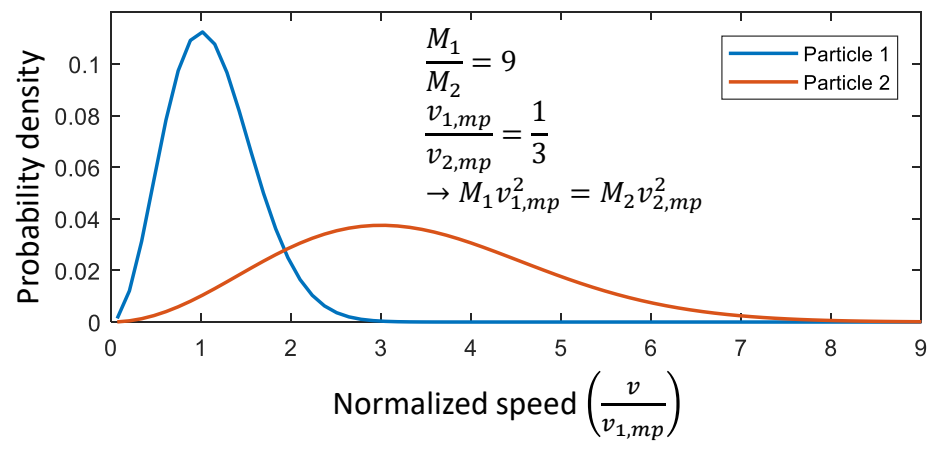

Figure 1. Maxwell-Boltzmann speed distributions of two types of particles

Boltzmann (1872) tried to provide mechanical proof of the Maxwell-Boltzmann speed distribution in 1872 by formulating the following equation.

$$
d n=f(x, t) d x \cdot f\left(x^{\prime}, t\right) d x^{\prime} \cdot \psi\left(\xi ; x, x^{\prime}\right) d \xi
$$

where $f(x, t) d x$ is the number of particles with speeds between $x$ and $x+d x$, and similarly for $f\left(x^{\prime}, t\right) d x^{\prime}, d n$ is the number of particles with speeds between $\xi$ and $\xi+d \xi$. In addition, the symbol $\psi\left(\xi ; x, x^{\prime}\right)$ represents the probability density function (PDF) of the resulting speed after a collision between two particles. The definition is excellent and meaningful, but the method used to calculate this PDF $\psi$ has yet to be created. Following Boltzmann's work in 1872, we derived the PDF $\psi$ based on Newton's law of motion in this paper. The PDF $\psi$ for equal mass particles had been provided by Lin et al. (2019).

To consider the collisions of unequal mass particles, we need to have four PDFs: $\psi^{11}\left(u_{1} ; v_{1}, v_{1}^{\prime}\right), \psi^{12}\left(u_{1} ; v_{1}, v_{2}\right)$, $\psi^{21}\left(u_{2} ; v_{2}, v_{1}\right)$, and $\psi^{22}\left(u_{2} ; v_{2}, v_{2}^{\prime}\right)$. Where $\psi^{a b}\left(u_{a} ; v_{a}, v_{b}\right)$ is the PDF of post-collision speed $u_{a}$ of a particle with a mass $M_{a}$ after the collision of two particles with mass $M_{a}$ in a pre-collision speed $v_{a}$ and mass $M_{b}$ in a 
pre-collision speed $v_{b}$. For $a=b$, the PDF is identical to the collision of two equal mass particles (Lin et al., 2019). For $a \neq b$, the PDF will be given in this paper.

After the PDF $\psi^{a b}\left(u_{a} ; v_{a}, v_{b}\right)$ was derived, a numerical iteration method (Lin et al., 2019) can be used to get a new distribution $\mathrm{P}_{\text {new }}^{a}\left(u_{a}\right)$ from the old distribution $\mathrm{P}_{\text {old }}^{a}\left(v_{a}\right)$, and set $\mathrm{P}_{\text {old }}^{a}\left(v_{a}\right)=\mathrm{P}_{\text {new }}^{a}\left(v_{a}\right)$ for the next iteration using the following equations.

$$
\mathrm{P}_{n e w}^{a}\left(u_{a}\right)=\sum_{b=1}^{N} \mathrm{n}_{b} \int_{0}^{\infty} \int_{0}^{\infty} \psi^{a b}\left(u_{a} ; v_{a}, v_{b}\right) \mathrm{P}_{o l d}^{a}\left(v_{a}\right) \mathrm{P}_{o l d}^{b}\left(v_{b}\right) d v_{a} d v_{b}, \quad a=1,2, \ldots \mathrm{N}
$$

Where $\mathrm{n}_{a}$ is the fraction of the number of particles with mass $M_{a}, \mathrm{~N}$ is the total number of particle types, and $\sum_{b=1}^{N} \mathrm{n}_{b}=1$.

Due to finite precision, the limit of computer memory, and computation time, the numerical iterations method can only apply to mixtures of gasses with a mass ratio between 0.01 and 100. In the cases of mixtures of molecules and subatomic particles, the extreme mass ratio is between $10^{-12}$ and $10^{12}$. This paper provides an analytical integration method to show that the Maxwell-Boltzmann speed distribution is valid for even these extreme cases. When $\mathrm{P}_{\text {old }}^{a}\left(v_{a}\right)$ and $\mathrm{P}_{\text {old }}^{b}\left(v_{b}\right)$ are the Maxwell-Boltzmann speed distributions, the analytical integration $\mathrm{P}_{\text {new }}^{a}\left(u_{a}\right)$ obtained by the following equation will also be the Maxwell-Boltzmann speed distribution.

$$
\mathrm{P}_{\text {new }}^{a}\left(u_{a}\right)=\int_{0}^{\infty} \int_{0}^{\infty} \psi^{a b}\left(u_{a} ; v_{a}, v_{b}\right) \mathrm{P}_{\text {old }}^{a}\left(v_{a}\right) \mathrm{P}_{\text {old }}^{b}\left(v_{b}\right) \mathrm{d} v_{a} \mathrm{~d} v_{b}
$$

The integration is tedious, but the final result is exactly the Maxwell-Boltzmann speed distribution. Moreover, the RMS speed square is inversely proportional to the particle masses as predicted by Avogadro's law (Avogadro).

\section{Velocity Diagram for a Collision of Two Particles}

A velocity diagram for a collision of two particles is used to derive the PDF of two types of particles' post-collision speed. Two concentric circles in the 2D plane can be constructed as a velocity diagram for the collision of two particles in 3D space. The concentric circles velocity diagram provides a geometric relationship between the pre-collision and post-collision speeds. The concentric circles velocity diagram was used by Maxwell in his study of the Maxwell-Boltzmann speed distribution and is explained and proved in this section.

Concentric circles velocity diagrams are based on two reference frames: the fixed reference frame $(\mathrm{O})$ and a center-of-mass $(\mathrm{CM})$ reference frame $(\mathrm{C})$. Speeds can be transferred between the fixed frame $(\mathrm{O})$ and the $\mathrm{CM}$ frame (C).

\subsection{The Fixed Reference Frame}

In the fixed reference frame, before a collision, two particles with mass $M_{1}$ and $M_{2}$ are moving at pre-collision velocities $\vec{v}_{1}$ (or $\overrightarrow{O A}$ ) and $\vec{v}_{2}$ (or $\overrightarrow{O B}$ ). After the collision, the post-collision velocities of the two particles change to $\vec{u}_{1}$ (or $\overrightarrow{O P}$ ) and $\vec{u}_{2}$ (or $\overrightarrow{O Q}$ ), as shown in the figure below. It is important to note that the variables in the PDF $\psi^{12}\left(u_{1} ; v_{1}, v_{2}\right)$ are speeds, which are the magnitudes of the velocities. Note that the vector $\vec{v}$ without an arrow-hat $v$ indicates the length of the vector. For example, $u_{1}=\left|\vec{u}_{1}\right|$.

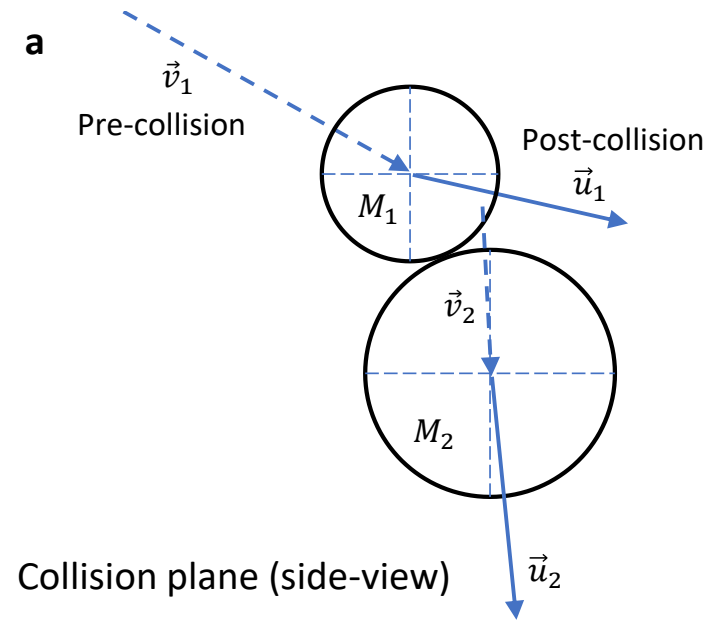

\section{b}

\section{Fixed frame (O)}

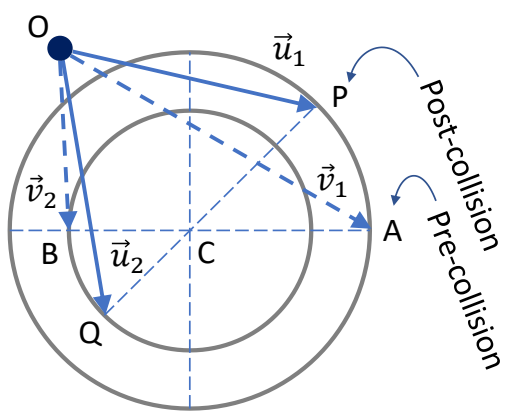

Concentric circles diagram

Figure 2. Velocities of two particles before and after a collision

\subsection{The Center-of-mass Reference Frame}

The center-of-mass (CM) reference frame uses the center of mass of two particles as its origin point (C). The velocity of the center-of-mass of two particles is related to the two masses $M_{1}$ and $M_{2}$ and their corresponding velocities $\vec{v}_{1}$ and 
$\vec{v}_{2}$ as

$$
\vec{v}_{c} \equiv \frac{M_{1} \vec{v}_{1}+M_{2} \vec{v}_{2}}{M_{1}+M_{2}} \equiv m_{1} \vec{v}_{1}+m_{2} \vec{v}_{2}
$$

where $m_{1}$ and $m_{2}$ are mass ratio and are defined as: $m_{1} \equiv \frac{M_{1}}{M_{1}+M_{2}} ; m_{2} \equiv \frac{M_{2}}{M_{1}+M_{2}}$.

Before the collision, the pre-collision velocities of particles 1 and 2 in the $\mathrm{CM}$ frame are

$$
\begin{aligned}
& \vec{v}_{1 c} \equiv \vec{v}_{1}-\vec{v}_{c} \\
& \vec{v}_{2 c} \equiv \vec{v}_{2}-\vec{v}_{c}
\end{aligned}
$$

It can be shown that after the collision, the post-collision velocities of particle 1 and particle 2 change to $\vec{u}_{1 c}$ (or $\overrightarrow{C P}$ ) and $\vec{u}_{2 c}$ (or $\overrightarrow{C Q}$ ) according to the following first three rules, which satisfy the last two conservations of momentum and energy.

1. Point $\mathrm{P}$ will be on Circle C-A. $\therefore u_{1 c}=v_{1 c}$

2. Point $Q$ will be on Circle C-B. $\therefore u_{2 c}=v_{2 c}$ and $\frac{u_{1 c}}{u_{2 c}}=\frac{|\overrightarrow{C P}|}{|\overrightarrow{C Q}|}=\frac{|\overrightarrow{C A}|}{|\overrightarrow{C B}|}=\frac{m_{2}}{m_{1}}$

3. Velocity $\overrightarrow{C P}$ and $\overrightarrow{C Q}$ have opposite directions. $\therefore m_{1} \vec{u}_{1 c}+m_{2} \vec{u}_{2 c}=0$

4. Conservation of momentum.

$$
\mathrm{m}_{1}\left(\vec{v}_{c}+\vec{u}_{1 c}\right)+\mathrm{m}_{2}\left(\vec{v}_{c}+\vec{u}_{2 c}\right)=\vec{v}_{c}=\mathrm{m}_{1}\left(\vec{v}_{c}+\vec{v}_{1 c}\right)+\mathrm{m}_{2}\left(\vec{v}_{c}+\vec{v}_{2 c}\right)
$$

5. Conservation of energy (by $u_{1 c}=v_{1 c}$ and $u_{2 c}=v_{2 c}$ ).

$$
\begin{array}{r}
\mathrm{m}_{1}\left(\vec{v}_{c}+\vec{u}_{1 c}\right) \cdot\left(\vec{v}_{c}+\vec{u}_{1 c}\right)+\mathrm{m}_{2}\left(\vec{v}_{c}+\vec{u}_{2 c}\right) \cdot\left(\vec{v}_{c}+\vec{u}_{2 c}\right)=v_{c}^{2}+\mathrm{m}_{1} u_{1 c}^{2}+\mathrm{m}_{2} u_{2 c}^{2} \\
\mathrm{~m}_{1}\left(\vec{v}_{c}+\vec{v}_{1 c}\right) \cdot\left(\vec{v}_{c}+\vec{v}_{1 c}\right)+\mathrm{m}_{2}\left(\vec{v}_{c}+\vec{v}_{2 c}\right) \cdot\left(\vec{v}_{c}+\vec{v}_{2 c}\right)=v_{c}^{2}+\mathrm{m}_{1} v_{1 c}^{2}+\mathrm{m}_{2} v_{2 c}^{2}
\end{array}
$$

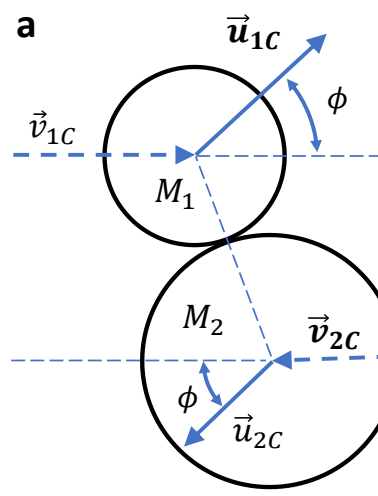

$$
\frac{u_{1 c}}{u_{2 c}}=\frac{v_{1 c}}{v_{2 c}}=\frac{\overline{C A}}{\overline{B C}}=\frac{m_{2}}{m_{1}}
$$

\section{Concentric circles diagram}

\section{Collision plane (side-view)}

b

Center-of-mass frame (C)

Figure 3. Post-collision velocities of two particles relative to the center of mass

\section{Probability Density Function of the Post-Collision Speed in Integral Form}

After any collision between two particles, the resulting speeds depend on two random factors: (1) the random directions of the pre-collision velocities $\vec{v}_{1}$ relative to $\vec{v}_{2}$ represented by a random angle $\alpha$ (see Figure 4), and (2) the random direction of the post-collision velocity $\vec{u}_{1}$ represented by a random angle $\beta$ (see Figure 4 ). These two random factors are discussed below to prepare for the derivation of the PDF $\psi^{12}\left(u_{1} ; v_{1}, v_{2}\right)$.

$$
\Psi^{12}\left(u_{1} ; v_{1}, v_{2}\right)=\int_{0}^{\pi} \mathrm{P}_{u_{1} \mid \alpha}\left(u_{1}\right) \mathrm{P}_{\alpha}(\alpha) d \alpha=\int_{0}^{\pi} \mathrm{P}_{\beta \mid \alpha}(\beta)\left|\frac{d \beta}{d u_{1}}\right| \mathrm{P}_{\alpha}(\alpha) d \alpha
$$

The right-hand side of the above equation will be derived in the following sections. And, the final PDF $\Psi^{12}$ will be obtained at the end of this section. 


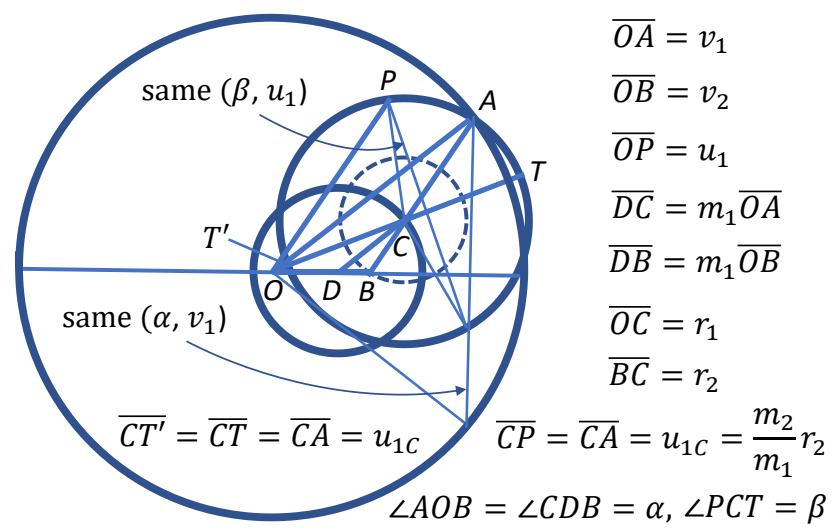

Figure 4. Three sphere surfaces $O-A, D-C, C$ - $P$ with centers at $O, D, C$, and radius $v_{1}, m_{1} v_{1}, \frac{m_{2}}{m_{1}} r_{2}$

\subsection{The Randomness of the Directions in 3D Before the Collision}

For two given pre-collision speeds $v_{1}$ and $v_{2}$, the post-collision speeds depend on the directions of the two pre-collision velocities $\vec{v}_{1}$ and $\vec{v}_{2}$. The two pre-collision velocities both have random directions. The directions of these two pre-collision velocities determine the radius of circle $C-A$ and circle $C$ - $B$. The randomness of the two velocities can be reduced to one random angle $\alpha$ which considers only the relative direction between $\vec{v}_{1}$ and $\vec{v}_{2}$ and is defined as the angle between the two pre-collision velocities $\vec{v}_{1}$ and $\vec{v}_{2}$ (in the fixed frame) as shown in Figure 4.

For fixed magnitudes of $v_{1}$ and $v_{2}$, if $\vec{v}_{2}=\overline{O B}$ is also fixed in the direction, but the direction of $\vec{v}_{1}=\overline{O A}$ is changed, then point $A$ will be located on a spherical surface, as shown in Figure 4. And the probability of point $A$ on the surface is uniformly distributed since $\vec{v}_{1}$ has equal opportunity in any direction. Therefore the probability density of point A located on the sphere surface $O-A$ at angle $\alpha$ is

$$
\mathrm{P}_{\alpha}(\alpha)=\frac{1}{2} \sin \alpha
$$

\subsection{The Randomness of the Directions in 3D after the Collision}

Similar to the pre-collision directions defined by $\alpha$ (representing the relative moving direction before the collision), the location of the point $\mathrm{P}$ will be defined by $\beta$, as the angle between $\vec{v}_{C}=\overline{O C}$ and $\vec{u}_{1 C}=\overline{C P}$, as shown in Figure 4 , such that the same angle $\beta$ will result in the same magnitude $u_{1}$ of the different velocity $\vec{u}_{1}$.

The band area between $\beta$ and $\beta+\mathrm{d} \beta$ on the sphere surface $C$ - $P$ is $2 \pi\left(u_{1 C} \sin \beta\right)\left(u_{1 C} \mathrm{~d} \beta\right)$, the total area of the sphere surface is $4 \pi u_{1 C}^{2}$, and the ratio is $\frac{1}{2} \sin \beta d \beta$. Therefore the probability density of point P located on the sphere surface $C$ - $P$ at angle $\beta$ is

$$
\mathrm{P}_{\beta \mid \alpha}(\beta)=\frac{1}{2} \sin \beta
$$

\subsection{Considering all the Possible Directions in 3D Before and after Collisions}

For a fixed $\alpha$, the $r_{1}$ and $r_{2}$ as shown in Figure 4, can be computed as

$$
\begin{aligned}
r_{1}\left(\alpha ; v_{1}, v_{2}\right) & =\overline{O C}=\sqrt{\left(m_{1} v_{1} \cos \alpha+m_{2} v_{2}\right)^{2}+\left(m_{1} v_{1} \sin \alpha\right)^{2}} \\
= & m_{1} \sqrt{v_{1}^{2}+\left(\frac{m_{2}}{m_{1}} v_{2}\right)^{2}+2 v_{1}\left(\frac{m_{2}}{m_{1}} v_{2}\right) \cos \alpha} \\
r_{2}\left(\alpha ; v_{1}, v_{2}\right) & =\overline{B C}=\sqrt{\left(m_{1} v_{1} \cos \alpha-m_{1} v_{2}\right)^{2}+\left(m_{1} v_{1} \sin \alpha\right)^{2}} \\
= & m_{1} \sqrt{v_{1}^{2}+v_{2}^{2}-2 v_{1} v_{2} \cos \alpha}
\end{aligned}
$$

So the relation between $u_{1}$ and $\beta$ for fixed $r_{1}$ and $r_{2}$ is given by 


$$
\begin{aligned}
u_{1}\left(\beta ; r_{1}, r_{2}\right)=\overline{O P} & =\sqrt{\left(\frac{m_{2}}{m_{1}} r_{2} \cos \beta+r_{1}\right)^{2}+\left(\frac{m_{2}}{m_{1}} r_{2} \sin \beta\right)^{2}} \\
& =\sqrt{r_{1}^{2}+\left(\frac{m_{2}}{m_{1}} r_{2}\right)^{2}+2 r_{1}\left(\frac{m_{2}}{m_{1}} r_{2}\right) \cos \beta}
\end{aligned}
$$

Hence

$$
\frac{d u_{1}}{d \beta}=\frac{-r_{1}\left(\frac{m_{2}}{m_{1}} r_{2}\right) \sin \beta}{\sqrt{r_{1}^{2}+\left(\frac{m_{2}}{m_{1}} r_{2}\right)^{2}+2 r_{1}\left(\frac{m_{2}}{m_{1}} r_{2}\right) \cos \beta}}=\frac{-r_{1}\left(\frac{m_{2}}{m} r_{2}\right) \sin \beta}{u_{1}}
$$

\subsection{Probability Density Function $\psi^{12}$ in Integral Form}

The PDF $\psi^{12}\left(u_{1} ; v_{1}, v_{2}\right)$ of the post-collision speed $u_{1}$ for two given pre-collision speeds $v_{1}$ and $v_{2}$ can be obtained by summating all densities for all possible directions, i.e., $\alpha$ between 0 and $\pi$, yields

$$
\begin{gathered}
\Psi^{12}\left(u_{1} ; v_{1}, v_{2}\right)=\int_{0}^{\pi} \mathrm{P}_{\beta \mid \alpha}(\beta)\left|\frac{d \beta}{d u_{1}}\right| \mathrm{P}_{\alpha}(\alpha) d \alpha=\int_{0}^{\pi} \frac{u_{1}}{4 r_{1}\left(\frac{m_{2}}{m_{1}} r_{2}\right)} \sin \alpha d \alpha \\
=\int_{0}^{\pi} \frac{u_{1} \sin \alpha d \alpha}{4 m_{1} m_{2} \sqrt{v_{1}^{2}+\left(\frac{m_{2}}{m_{1}} v_{2}\right)^{2}+2 v_{1}\left(\frac{m_{2}}{m_{1}} v_{2}\right) \cos \alpha \sqrt{v_{1}^{2}+v_{2}^{2}-2 v_{1} v_{2} \cos \alpha}}} \\
=\frac{u_{1}}{4 m_{1} m_{2}} \int_{0}^{\pi} \frac{\sin \alpha d \alpha}{\sqrt{C+2 B \cos \alpha-A \cos ^{2} \alpha}}
\end{gathered}
$$

Where

$$
\begin{aligned}
& A=4 v_{1}^{2} \hat{v}_{2}^{2}=r^{4} \sin ^{2}(2 \theta) \\
& B=v_{1}\left(\frac{m_{2}}{m_{1}} v_{2}\right)\left(v_{1}^{2}+v_{2}^{2}\right)-v_{1} v_{2}\left[v_{1}^{2}+\left(\frac{m_{2}}{m_{1}} v_{2}\right)^{2}\right] \\
& =2 v_{1} \hat{v}_{2}\left(v_{1}^{2}-\hat{v}_{2}^{2}\right)\left(\frac{m_{2}-m_{1}}{\sqrt{4 m_{1} m_{2}}}\right)=r^{4} \sin (2 \theta) \cos (2 \theta) \tan \varphi \\
& C=\left[v_{1}^{2}+\left(\frac{m_{2}}{m_{1}} v_{2}\right)^{2}\right]\left(v_{1}^{2}+v_{2}^{2}\right) \\
& \sqrt{B^{2}+A C}=v_{1}\left(\frac{m_{2}}{m_{1}} v_{2}\right)\left(v_{1}^{2}+v_{2}^{2}\right)+v_{1} v_{2}\left[v_{1}^{2}+\left(\frac{m_{2}}{m_{1}} v_{2}\right)^{2}\right] \\
& =2 v_{1} \hat{v}_{2}\left(v_{1}^{2}+\hat{v}_{2}^{2}\right)\left(\frac{1}{\sqrt{4 m_{1} m_{2}}}\right)=r^{4} \sin (2 \theta) / \cos \varphi \\
& \hat{v}_{2}=\sqrt{\frac{m_{2}}{m_{1}}} v_{2}, \quad r=\sqrt{v_{1}^{2}+\hat{v}_{2}^{2}}, \quad \theta=\tan ^{-1}\left(\frac{\hat{v}_{2}}{v_{1}}\right) \\
& \varphi=\tan ^{-1}\left(\frac{m_{2}-m_{1}}{\sqrt{4 m_{1} m_{2}}}\right), \quad m_{1}=\frac{M_{1}}{M_{1}+M_{2}}, \quad m_{2}=\frac{M_{2}}{M_{1}+M_{2}}
\end{aligned}
$$

\section{Probability Density Function of the Post-Collision Speed in Evaluated Form}

The PDF $\psi^{12}$, shown in Equation (17), is in integral form and can be used to compute numerically the probability of post-collision speed of particles 1 after a collision. Because the PDF in integral form can be computed numerically, it can be used in Equation (8) to mechanical proof the Maxwell-Boltzmann speed distribution using numerical iteration. The limitation of computer-aided proof of the Maxwell-Boltzmann speed distribution is that two particles' mass ratio cannot be near infinite. 
In order for the PDF $\psi^{12}$ to be used for analytical proof of the Maxwell-Boltzmann speed distribution, the PDF $\Psi^{12}$ must be evaluated and formulated for all the possible pre-collision speeds of the two particles as detailed in the following sections.

\subsection{Integrate the Probability Density Function}

The PDF of the post-collision speed in the previous section is in integral form with an interval from 0 to $\pi$. Since some $\alpha$ values in the interval near the lower bound $\alpha=0$ and the upper bound $\alpha=\pi$ cannot have a valid real number but some imaginary number, it is required to use the proper interval $\left(\alpha_{l b}, \alpha_{u b}\right)$ of the integration and integrate the PDF $\psi^{12}$ of Equation (17) as

$$
\begin{gathered}
\Psi^{12}\left(u_{1} ; v_{1}, v_{2}\right)=\frac{u_{1}}{4 m_{1} m_{2}} \int_{\alpha_{l b}}^{\alpha_{u b}} \frac{\sin \alpha d \alpha}{\sqrt{C+2 B \cos \alpha-A \cos ^{2} \alpha}} \\
=\frac{u_{1}}{4 m_{1} m_{2} \sqrt{A}}\left[\sin ^{-1} \frac{B-A \cos \alpha}{\sqrt{B^{2}+A C}}\right]_{\alpha_{l b}}^{\alpha_{u b}} \\
=\frac{u_{1}}{4 m_{1} m_{2} r^{2} \sin (2 \theta)}\left[\sin ^{-1}(\sin \varphi \cos (2 \theta)-\cos \varphi \sin (2 \theta) \cos \alpha)\right]_{\alpha_{l b}}^{\alpha_{u b}} \\
=\frac{u_{1}\left(\gamma_{u b}-\gamma_{l b}\right)}{4 m_{1} m_{2} r^{2} \sin (2 \theta)}
\end{gathered}
$$

where

$$
\begin{aligned}
& \gamma_{l b, u b}=\sin ^{-1}\left(\sin \varphi \cos (2 \theta)-\cos \varphi \sin (2 \theta) \cos \alpha_{l b, u b}\right) \\
& r=\sqrt{v_{1}^{2}+\hat{v}_{2}^{2}}, \theta=\tan ^{-1}\left(\frac{\hat{v}_{2}}{v_{1}}\right), \varphi=\tan ^{-1}\left(\frac{m_{2}-m_{1}}{\sqrt{4 m_{1} m_{2}}}\right)
\end{aligned}
$$

\subsection{Determine the Bounds of Interval}

We will determine the lower bound $\left(\alpha_{l b}\right)$ and upper bound $\left(\alpha_{u b}\right)$ of $\angle A O B(\alpha)$ for given pre-collision speed $\left(v_{1}\right)$, pre-collision speed $\left(v_{2}\right)$ and a target post-collision speed $\left(u_{1}\right)$ in the above $\operatorname{PDF} \psi^{12}\left(u_{1} ; v_{1}, v_{2}\right)$. The $\angle A O B(\alpha)$ is the angle between the pre-collision speed $\left(v_{1}\right)$ and pre-collision speed $\left(v_{2}\right)$, as shown in Figure 4 .

The possible range of $\angle A O B(\alpha)$ is from 0 to $\pi$. However, some ranges of $\alpha(\angle A O B)$, near 0 or/and $\pi$, are impossible to reach the target post-collision speed $\left(u_{1}\right)$ because $u_{1}(\overline{O P})$ is bound by $\overline{O T^{\prime}}$ and $\overline{O T}$ as following

$$
\overline{O T^{\prime}} \leq \overline{O P} \leq \overline{O T}
$$

Where

$$
\begin{gathered}
\overline{O P}=u_{1} \\
\overline{O T^{\prime}}=\left|r_{1}\left(\alpha ; v_{1}, v_{2}\right)-\frac{m_{2}}{m_{1}} r_{2}\left(\alpha ; v_{1}, v_{2}\right)\right| \\
\overline{O T}=r_{1}\left(\alpha ; v_{1}, v_{2}\right)+\frac{m_{2}}{m_{1}} r_{2}\left(\alpha ; v_{1}, v_{2}\right)
\end{gathered}
$$

Where, $r_{1}$ and $r_{2}$ as shown in Figure 4, can be computed from $\alpha$ by Equation (14).

The inequation of Equation (21) can be formulated in terms of $v_{1}, v_{2}, u_{1}, \alpha$ and separated into two inequations as

$$
\begin{aligned}
& \left|m_{1} \sqrt{v_{1}^{2}+\left(\frac{m_{2}}{m_{1}} v_{2}\right)^{2}+2 v_{1}\left(\frac{m_{2}}{m_{1}} v_{2}\right) \cos \alpha}-m_{2} \sqrt{v_{1}^{2}+v_{2}^{2}-2 v_{1} v_{2} \cos \alpha}\right| \leq u_{1} \\
& u_{1} \leq m_{1} \sqrt{v_{1}^{2}+\left(\frac{m_{2}}{m_{1}} v_{2}\right)^{2}+2 v_{1}\left(\frac{m_{2}}{m_{1}} v_{2}\right) \cos \alpha}+m_{2} \sqrt{v_{1}^{2}+v_{2}^{2}-2 v_{1} v_{2} \cos \alpha}
\end{aligned}
$$

Let the $\alpha$ in lower bound and upper bound of $u_{1}$ be $\alpha_{u b}$ and $\alpha_{l b}$ respectively and express the boundary values of the above equation using a plus-minus sign in conjunction with $\alpha_{l b, u b}$ for a more compact formulation as

$$
u_{1}=\left|m_{1} \sqrt{v_{1}^{2}+\left(\frac{m_{2}}{m_{1}} v_{2}\right)^{2}+2 v_{1}\left(\frac{m_{2}}{m_{1}} v_{2}\right) \cos \alpha_{l b, u b}} \pm m_{2} \sqrt{v_{1}^{2}+v_{2}^{2}-2 v_{1} v_{2} \cos \alpha_{l b, u b}}\right|
$$

The $\cos \alpha_{l b}$ and $\cos \alpha_{u b}$ in the above equation can be solved by taking square twice (Note 1), as shown in Equation (25). For convenience, let us assign a new variable $c_{l b, u b}$ to the solutions of $\cos \alpha_{l b, u b}$ as 


$$
\cos \alpha_{l b, u b}=\frac{\left(m_{2}-m_{1}\right)\left(v_{1}^{2}-u_{1}^{2}\right) \pm \sqrt{4 m_{1} m_{2}} u_{1} \sqrt{v_{1}^{2}+\hat{v}_{2}^{2}-u_{1}^{2}}}{\sqrt{4 m_{1} m_{2}} v_{1} \hat{v}_{2}} \equiv c_{l b, u b}
$$

Or in polar coordinate as follows, where $\theta_{o}=\cos ^{-1}\left(u_{1} / r\right)$.

$$
\cos \alpha_{l b, u b}=\frac{\sin \varphi \cos (2 \theta) \pm \sin \left(2 \theta_{o} \mp \varphi\right)}{\cos \varphi \sin (2 \theta)} \equiv c_{l b, u b}
$$

Because the value of any cosine function cannot be smaller than -1 or larger than +1 , we must limit the values of $\cos \alpha_{l b, u b}$ between -1 and +1 as

$$
\cos \alpha_{l b}=\min \left(c_{l b},+1\right) \quad \text { and } \quad \cos \alpha_{u b}=\max \left(c_{u b},-1\right)
$$

In the table below, $\gamma_{l b, u b}$ for each case is calculated by Equation (20a) as follows.

For Case 1 and Case 3, $\left|c_{l b, u b}\right|>1: \cos \alpha_{l b, u b}= \pm 1 \quad\left(\alpha_{l b}=0, \alpha_{u b}=\pi\right)$

$$
\begin{gathered}
\gamma_{l b, u b}=\sin ^{-1}(\sin \varphi \cos (2 \theta) \mp \cos \varphi \sin (2 \theta))=\mp \sin ^{-1}(\sin (2 \theta \mp \varphi)) \\
=\mp \min ((2 \theta \mp \varphi),(\pi-2 \theta \pm \varphi)) \in[-\pi / 2, \pi / 2]
\end{gathered}
$$

For Case 2 and Case 4, $\left|c_{l b, u b}\right| \leq 1: \cos \alpha_{l b, u b}=c_{l b, u b} \quad\left(\alpha_{l b, u b}=\cos ^{-1} c_{l b, u b}\right)$

$$
\begin{aligned}
& \gamma_{l b, u b}= \sin ^{-1}\left(\sin \varphi \cos (2 \theta)-\sin \varphi \cos (2 \theta) \mp \sin \left(2 \theta_{o} \mp \varphi\right)\right) \\
&=\mp \sin ^{-1}\left(\sin \left(2 \theta_{o} \mp \varphi\right)\right) \\
&=\mp \min \left(\left(2 \theta_{0} \mp \varphi\right),\left(\pi-2 \theta_{0} \pm \varphi\right)\right) \epsilon[-\pi / 2, \pi / 2]
\end{aligned}
$$

\begin{tabular}{|c|c|c|c|c|c|}
\hline & IF & Use & $\alpha$ & $\gamma$ & \\
\hline Case 1 & $c_{l b}>1$ & $c_{l b}=\cos \left(\alpha_{l b}\right)=1$ & $\alpha_{l b}=0$ & $\gamma_{l b}=-\min [(2 \theta-\varphi),(\pi-2 \theta+\varphi)]^{*}$ & \multirow{3}{*}{$\begin{array}{l}4 \text { Possible } \\
\text { L bounds } \\
4 \text { Possible }\end{array}$} \\
\hline Case 2 & $c_{l b}<1$ & \multicolumn{2}{|c|}{$\alpha_{l b}=\cos ^{-1}\left(c_{l b}\right)$} & $\gamma_{l b}=-\min \left[\left(2 \theta_{0}-\varphi\right),\left(\pi-2 \theta_{0}+\varphi\right)\right]^{*}$ & \\
\hline Case 3 & $c_{u b}<-1$ & $c_{u b}=\cos \left(\alpha_{u b}\right)=-1$ & $\alpha_{u b}=\pi$ & $\gamma_{u b}=\min [(2 \theta+\varphi),(\pi-2 \theta-\varphi)] * *$ & \\
\hline Case 4 & $c_{u b}>-1$ & \multicolumn{2}{|c|}{$\alpha_{u b}=\cos ^{-1}\left(c_{u b}\right)$} & $\gamma_{u b}=\min \left[\left(2 \theta_{0}+\varphi\right),\left(\pi-2 \theta_{0}-\varphi\right)\right]^{* *}$ & U bounds \\
\hline
\end{tabular}

Equation (27) and Equation (28) can be combined to

$$
\gamma_{l b, u b}=\mp \min \left((2 \theta \mp \varphi),(\pi-2 \theta \pm \varphi),\left(2 \theta_{0} \mp \varphi\right),\left(\pi-2 \theta_{0} \pm \varphi\right)\right)
$$

Table 1. The functions $\gamma_{l b}$ and $\gamma_{u b}$ for all cases

Now we have determined the bounds $\alpha_{l b, u b}$ of the integral and the values $\gamma_{l b, u b}$. Let's summarize the PDF again as following

$$
\Psi^{12}\left(u_{1} ; v_{1}, v_{2}\right)=\frac{u_{1}\left(\gamma_{u b}-\gamma_{l b}\right)}{8 m_{1} m_{2} v_{1} \hat{v}_{2}}
$$

where

$$
\begin{gathered}
\gamma_{l b, u b}=\mp \min \left((2 \theta \mp \varphi),(\pi-2 \theta \pm \varphi),\left(2 \theta_{0} \mp \varphi\right),\left(\pi-2 \theta_{0} \pm \varphi\right)\right) \\
\hat{v}_{2}=\sqrt{\frac{m_{2}}{m_{1}}} v_{2}, \quad \theta=\tan ^{-1}\left(\frac{\hat{v}_{2}}{v_{1}}\right), \quad \varphi=\tan ^{-1}\left(\frac{m_{2}-m_{1}}{\sqrt{4 m_{1} m_{2}}}\right) \\
r=\sqrt{v_{1}^{2}+\hat{v}_{2}^{2}}, \quad \theta_{o}=\cos ^{-1}\left(\frac{u_{1}}{r}\right)
\end{gathered}
$$



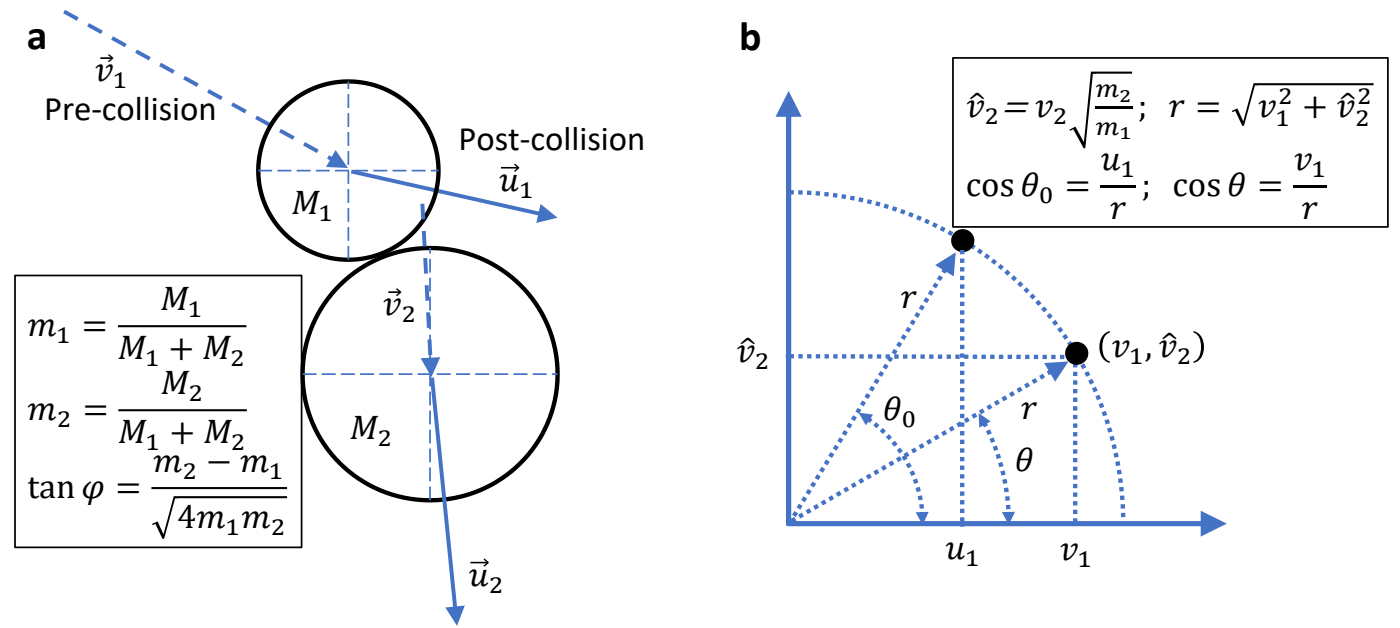

Figure 5. Relations between $\left(r, \theta, \theta_{0}\right)$ and $\left(u_{1}, v_{1}, \hat{v}_{2}\right)$

\subsection{Regions of $\left(\gamma_{u b}-\gamma_{l b}\right)$ by the Lower and Upper Bounds}

The PDF $\Psi^{12}$ in the equation above cannot be formulated with one formula but 16 formulas. Because $\gamma_{l b}$ has four possible formulas of $(2 \theta-\varphi),(\pi-2 \theta+\varphi),\left(2 \theta_{0}-\varphi\right),\left(\pi-2 \theta_{0}+\varphi\right)$, and $\gamma_{u b}$ have four possible formulas of $(2 \theta+\varphi),(\pi-2 \theta-\varphi),\left(2 \theta_{0}+\varphi\right),\left(\pi-2 \theta_{0}-\varphi\right)$, the total combination of $\left(\gamma_{u b}-\gamma_{l b}\right)$ is sixteen $(4 \times 4)$, as shown in the table below.

Table 2. Sixteen formulas of $\left(\gamma_{u b}-\gamma_{l b}\right)$ for computing $\psi^{12}\left(u_{1} ; v_{1}, v_{2}\right)$

\begin{tabular}{|c|c|c|c|c|c|}
\hline \multirow[b]{2}{*}{ Upper bound } & \multirow[t]{2}{*}{ Lower bound } & \multicolumn{2}{|c|}{$\begin{array}{l}\text { Case 1: } c_{l b}>1 \\
\quad \rightarrow \alpha_{l b}=0\end{array}$} & \multicolumn{2}{|c|}{$\begin{aligned} & \text { Case 2: } c_{l b}<1 \\
\rightarrow & \alpha_{l b}=\cos ^{-1}\left(c_{l b}\right)\end{aligned}$} \\
\hline & & $2 \theta-\varphi$ & $\pi-2 \theta+\varphi$ & $2 \theta_{0}-\varphi$ & $\pi-2 \theta_{0}+\varphi$ \\
\hline \multirow{2}{*}{$\begin{array}{c}\text { Case 3: } \\
\begin{array}{c}c_{u b}<-1 \\
\downarrow \\
\alpha_{u b}=\pi\end{array}\end{array}$} & $2 \theta+\varphi$ & $4 \theta$ & $\begin{array}{c}\text { B (D-J Boundary) } \\
\pi+2 \varphi\end{array}$ & $\begin{array}{c}\text { C (A-K Boundary) } \\
2 \theta+2 \theta_{0}\end{array}$ & $\begin{array}{c}\mathbf{D}\left(m_{1}>m_{2}\right) \\
\pi+2 \varphi \\
+2 \theta-2 \theta_{0} \\
\end{array}$ \\
\hline & $\pi-2 \theta-\varphi$ & $\begin{array}{c}\text { E (G-M Boundary) } \\
\pi-2 \varphi\end{array}$ & $2 \pi-4 \theta$ & $\begin{array}{c}\mathbf{G}\left(m_{1}<m_{2}\right) \\
\pi-2 \varphi \\
-2 \theta+2 \theta_{0}\end{array}$ & $\begin{array}{l}\text { H (F-P Boundary) } \\
\quad 2 \pi-2 \theta-2 \theta_{0}\end{array}$ \\
\hline \multirow{2}{*}{$\begin{array}{c}\text { Case 4: } \\
c_{u b}>-1 \\
\downarrow \\
\alpha_{u b}=\cos ^{-1}\left(c_{u b}\right)\end{array}$} & $2 \theta_{0}+\varphi$ & $\begin{array}{c}\text { I (A-K Boundary) } \\
2 \theta+2 \theta_{0}\end{array}$ & $\begin{array}{c}\mathbf{J}\left(m_{1}>m_{2}\right) \\
\pi+2 \varphi \\
-2 \theta+2 \theta_{0} \\
\end{array}$ & $4 \theta_{0}$ & $\begin{array}{c}\text { L (D-J Boundary) } \\
\pi+2 \varphi\end{array}$ \\
\hline & $\pi-2 \theta_{0}-\varphi$ & $\begin{array}{c}\mathbf{M}\left(m_{1}<m_{2}\right) \\
\pi-2 \varphi \\
+2 \theta-2 \theta_{0}\end{array}$ & $\begin{array}{l}\mathbf{N} \text { (F-P Boundary) } \\
2 \pi-2 \theta-2 \theta_{0}\end{array}$ & $\begin{array}{c}\text { O (G-M Boundary) } \\
\pi-2 \varphi\end{array}$ & $2 \pi-4 \theta_{0}$ \\
\hline
\end{tabular}

The formulas of $\left(\gamma_{u b}-\gamma_{l b}\right)$ in the table above can be visualized as eight regions (ADFGJKMP) with coordinates of $v_{1}$ and $\hat{v}_{2}$ as shown in Figure 6. Each region represents a formula of $\left(\gamma_{u b}-\gamma_{l b}\right)$. Both $\gamma_{u b}$ and $\gamma_{l b}$ have four possible formulas, as shown in Equation (29), which in terms depends on the pre-collision speeds of two particles before a collision. The formula of $\left(\gamma_{u b}-\gamma_{l b}\right)$ is the addition of $\gamma_{u b}$ (Case 3 and Case 4) and $-\gamma_{l b}$ (Case 1 and Case $2)$ as shown in the table above.

In Figure 6, eight formulas (BCEHILNO) are not shown as regions. It can easily be proved that these eight formulas (BCEHILNO) are the boundary between two regions. For example, Formula E or $\mathrm{O}(\pi-2 \varphi)$ is the boundary (part of $B L_{2}$ ) between Formula $\mathrm{G}$ and Formula $\mathrm{M}$ and can be obtained by equating the Formula $\mathrm{G}$ and Formula $\mathrm{M}$ as

$$
\pi-2 \varphi-2 \theta+2 \theta_{0}=\pi-2 \varphi+2 \theta-2 \theta_{0} \rightarrow \theta=\theta_{0} \rightarrow B L_{2}
$$

Substituting the above equation of the boundary line $\left(B L_{2}\right)$ into Formulas $\mathrm{G}$ and $\mathrm{M}$ shows that Formula $\mathrm{G}$ and Formula $\mathrm{M}$ merge to Formula $\mathrm{E}$ or $\mathrm{O}$ as

Formula G: $\pi-2 \varphi-2 \theta+2 \theta_{0} \rightarrow \pi-2 \varphi \rightarrow$ Formula E or O

Formula M: $\pi-2 \varphi+2 \theta-2 \theta_{0} \rightarrow \pi-2 \varphi \rightarrow$ Formula E or O

Other examples for the boundary lines $B L_{6}$ and $B L_{7}$ between Formula M-A and Formula M- 0 can be obtained by 
Formula $\mathrm{M}=$ Formula $\mathrm{A}$ and Formula $\mathrm{M}=0$ as

$$
\begin{gathered}
\pi-2 \varphi+2 \theta-2 \theta_{0}=4 \theta \rightarrow \theta=\pi / 2-\varphi-\theta_{0} \rightarrow B L_{6} \\
\pi-2 \varphi+2 \theta-2 \theta_{0}=0 \rightarrow \theta=-\left(\pi / 2-\varphi-\theta_{0}\right) \rightarrow B L_{7}
\end{gathered}
$$

All the boundary lines in Figure 6 can be found from the formulas of two adjacent regions as described above and are listed in the table below.

Table 3. Equations of the boundary lines

\begin{tabular}{|c|l|l|}
\hline Boundary line (BL) & Adjacent regions & Equation of boundary line \\
\hline$B L_{0}$ & K-0 & $\theta_{0}=0 ; r=u_{1}$ \\
\hline$B L_{1}$ & F-0 & $\theta=\pi / 2$ \\
\hline$B L_{2}$ & F-P; G-M; K-A & $\theta=\theta_{0} ; v_{1}=u_{1}$ \\
\hline$B L_{3}$ & A-0 & $\theta=0$ \\
\hline$B L_{4,8}$ & P-M; F-G, P-D; F-J & $\theta=\pi / 2 \pm \varphi-\theta_{0}$ \\
\hline$B L_{5,9}$ & G-0, J-0 & $\theta=\pi / 2 \mp \varphi+\theta_{0}$ \\
\hline$B L_{6,10}$ & G-K; M-A, J-K; D-A & $\theta=\pi / 2 \mp \varphi-\theta_{0}$ \\
\hline$B L_{7,11}$ & M-0, D-0 & $\theta=-\left(\pi / 2 \mp \varphi-\theta_{0}\right)$ \\
\hline
\end{tabular}

For the case of $m_{1} \leq m_{2}$, the angle $\varphi$ is greater than zero $\left(\varphi=\tan ^{-1}\left(\frac{m_{2}-m_{1}}{\sqrt{4 m_{1} m_{2}}}\right) \geq 0\right)$, and there are six regions (AFGKMP) of $\left(\gamma_{u b}-\gamma_{l b}\right)$, as shown in Figure 6(a). For the case of $m_{1} \geq m_{2}$, the angle $\varphi$ is less than zero ( $\varphi \leq 0$ ), and region $\mathrm{G}$ becomes Region $\mathrm{J}$, and region $\mathrm{M}$ becomes Region $\mathrm{D}$, as shown in Figure 6(b).

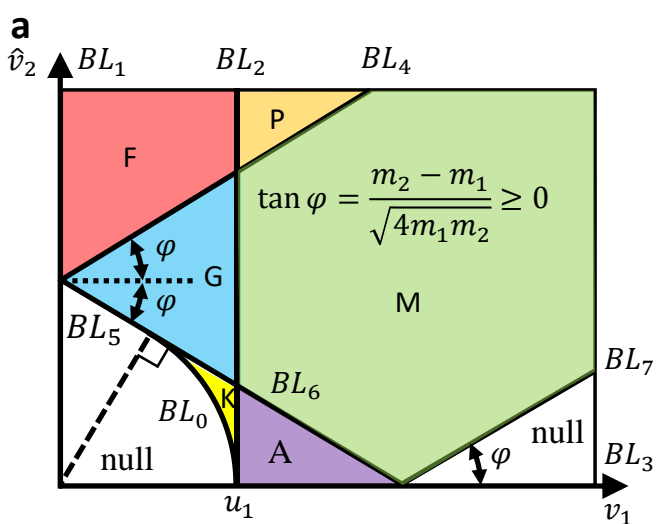

Regions and boundary lines for $m_{1} \leq m_{2}$

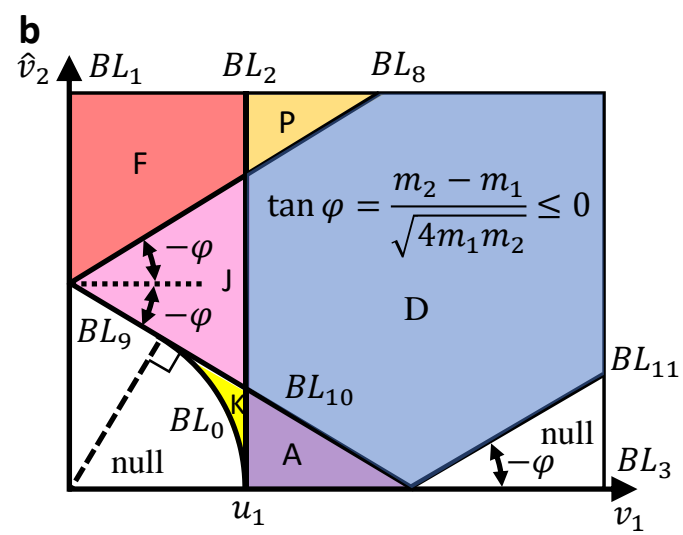

Regions and boundary lines for $m_{1} \geq m_{2}$

Figure 6. Eight regions of $\left(\gamma_{u b}-\gamma_{l b}\right)$ and boundary lines

As it can be observed in Figure 6, the shapes of regions of $\left(\gamma_{u b}-\gamma_{l b}\right)$ is related to the angle $\varphi$, which is defined as $\tan ^{-1}\left(\frac{m_{2}-m_{1}}{\sqrt{4 m_{1} m_{2}}}\right)$, as shown in Equation (18h). Four combinations of $m_{1}$ and $m_{2}$ are used as examples to visualize the regions of $\left(\gamma_{u b}-\gamma_{l b}\right)$ for some typical mass ratios. The four mass ratios are (a) $m_{1}=0.5, m_{2}=0.5$, (b) $m_{1}=$ $0.3, m_{2}=0.7$, (c) $m_{1}=0.1, m_{2}=0.9$, (d) $m_{1}=0.01, m_{2}=0.99$. Where (a) is a special case representing equal-mass particles. The angle $\varphi=\tan ^{-1}\left(\frac{m_{2}-m_{1}}{\sqrt{4 m_{1} m_{2}}}\right)$ determines the slope of the boundary lines $B L_{4}$ to $B L_{11}$ as shown in Figure 6 and Figure 7. 

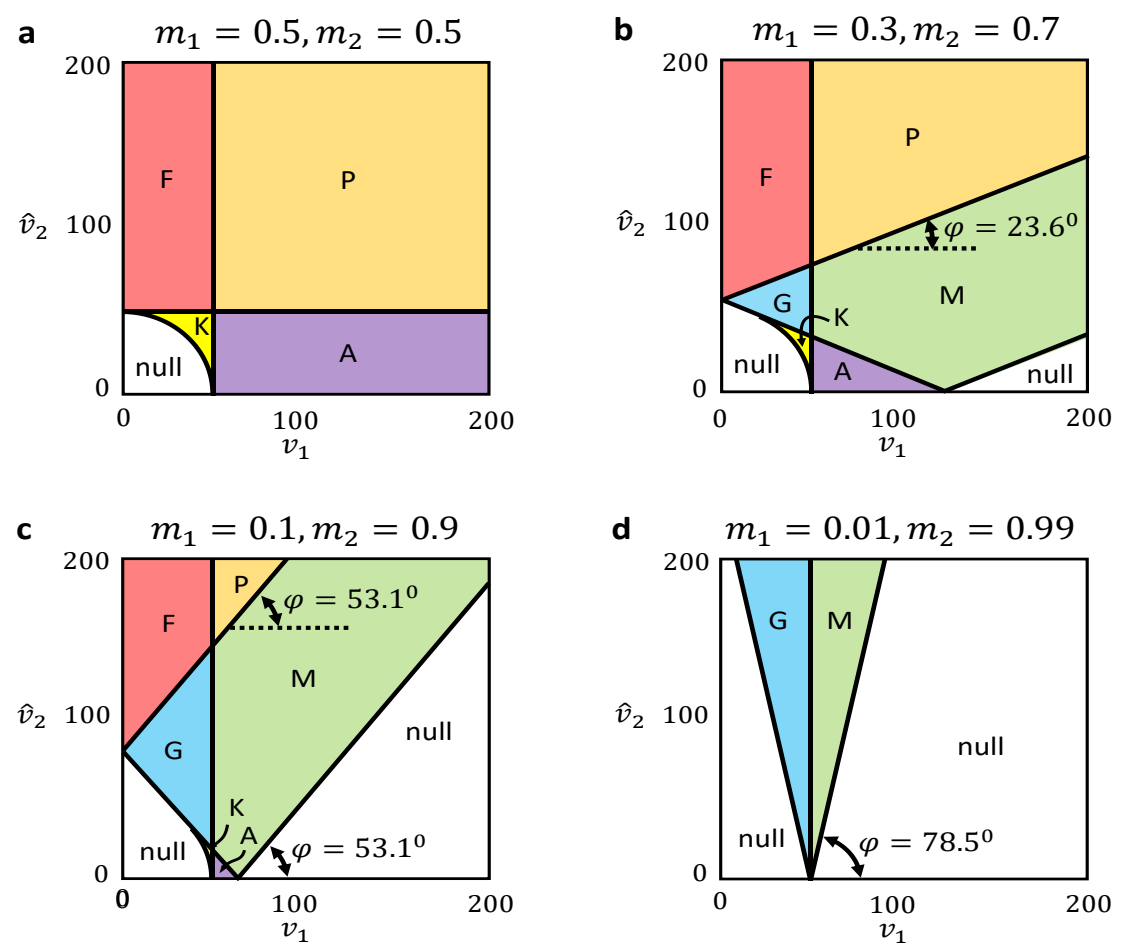

Figure 7. Regions A to $\mathrm{P}$ for the formulas of $\left(\gamma_{u b}-\gamma_{l b}\right)$

\section{Analytical Proof by Double Integrals}

for computing $\psi^{12}\left(u_{1} ; v_{1}, v_{2}\right)$ with $u_{1}=50$

The Maxwell-Boltzmann speed distribution is proved by examining speed distributions before and after a random collision of two particles in a steady-state system. We assumed the speed distributions of both particle 1 and particle 2 before a collision are the Maxwell-Boltzmann speed distributions. Suppose the integrated speed distributions of both particle 1 and particle 2 after the collision are also the Maxwell-Boltzmann speed distribution. In that case, it proves that the Maxwell-Boltzmann speed distribution is the right speed distribution of the steady-state system.

Before a random collision, we let the speed distributions $\mathrm{P}_{\text {old }}^{1}\left(v_{1}\right), \mathrm{P}_{\text {old }}^{2}\left(v_{2}\right)$ of particle 1 and particle 2 respectively be the Maxwell-Boltzmann speed distribution as

$$
\begin{aligned}
& \mathrm{P}_{\text {old }}^{1}\left(v_{1}\right)=\frac{4 h_{1}^{3}}{\sqrt{\pi}} v_{1}^{2} e^{-h_{1}^{2} v_{1}^{2}} \\
& \mathrm{P}_{\text {old }}^{2}\left(v_{2}\right)=\frac{4 h_{2}^{3}}{\sqrt{\pi}} v_{2}^{2} e^{-h_{2}^{2} v_{2}^{2}}
\end{aligned}
$$

Where $h_{1}=\sqrt{\frac{M_{1}}{2 k T}}, h_{2}=\sqrt{\frac{M_{2}}{2 k T}}$ and $k$ is the Boltzmann constant, $T$ is the temperature.

The speed distributions $\mathrm{P}_{\text {new }}^{1}\left(u_{1}\right)$ of particle 1 after the collision can be calculated from the following equation with the PDF $\psi^{12}\left(u_{1} ; v_{1}, v_{2}\right)$ computed by Equation (30) as

$$
\begin{aligned}
& \mathrm{P}_{\text {new }}^{1}\left(u_{1}\right)=\int_{0}^{\infty} \int_{0}^{\infty} \psi^{12}\left(u_{1} ; v_{1}, v_{2}\right) \mathrm{P}_{\text {old }}^{1}\left(v_{1}\right) \mathrm{P}_{\text {old }}^{2}\left(v_{2}\right) d v_{1} d v_{2} \\
& \quad=\int_{0}^{\infty} \int_{0}^{\infty} \psi^{12}\left(u_{1} ; v_{1}, v_{2}\right) \frac{4 h_{1}^{3}}{\sqrt{\pi}} v_{1}^{2} e^{-h_{1}^{2} v_{1}^{2}} \frac{4 h_{2}^{3}}{\sqrt{\pi}} v_{2}^{2} e^{-h_{2}^{2} v_{2}^{2}} d v_{1} d v_{2}
\end{aligned}
$$

Where $\psi^{12}\left(u_{1} ; v_{1}, v_{2}\right)$ is the PDF of post-collision speed $u_{1}$ of particle 1 after a random collision of particle 1 with pre-collision speed $v_{1}$ and particle 2 with pre-collision speed $v_{2}$ as

$$
\psi^{12}\left(u_{1} ; v_{1}, v_{2}\right)=\frac{\left(\gamma_{u b}-\gamma_{l b}\right) u_{1}}{8 m_{1} m_{2} v_{1} \hat{v}_{2}}
$$


with

$$
\left(\gamma_{u b}-\gamma_{l b}\right)=0, \quad \text { for } r \leq u_{1} \text { or } \gamma_{u b} \leq \gamma_{l b}
$$

\subsection{Simplify the Distribution Parameters}

To further evaluate the speed distribution $\mathrm{P}_{\text {new }}^{1}\left(u_{1}\right)$ as in Equation (33), the speed distribution can be first simplified by eliminating $h_{2}$ by the definition of the Boltzmann constant $k$ as shown below

$$
\frac{h_{2}}{h_{1}}=\frac{\sqrt{\frac{M_{2}}{2 k T}}}{\sqrt{\frac{M_{1}}{2 k T}}}=\sqrt{\frac{M_{2}}{M_{1}}}=\sqrt{\frac{m_{2}}{m_{1}}}
$$

Since $v_{2}=\sqrt{\frac{m_{1}}{m_{2}}} \hat{v}_{2}\left(\right.$ as defined in Equation (18e)) and simplified symbol $h_{1}$ as $h$ will yield

$$
\begin{gathered}
\mathrm{P}_{n e w}^{1}\left(u_{1}\right)=\int_{0}^{\infty} \int_{0}^{\infty} \psi^{12}\left(u_{1} ; v_{1}, v_{2}\right) \frac{4 h_{1}^{3}}{\sqrt{\pi}} v_{1}^{2} e^{-h_{1}^{2} v_{1}^{2}} \frac{4 h_{2}^{3}}{\sqrt{\pi}} v_{2}^{2} e^{-h_{2}^{2} v_{2}^{2}} d v_{1} d v_{2} \\
=\int_{0}^{\infty} \int_{0}^{\infty} \Psi^{12}\left(u_{1} ; v_{1}, v_{2}\right) \frac{16 h^{6}}{\pi} v_{1}^{2} \hat{v}_{2}^{2} e^{-h^{2}\left(v_{1}^{2}+\hat{v}_{2}^{2}\right)} d v_{1} d \hat{v}_{2} \\
=\frac{16 h^{6}}{\pi} \int_{0}^{\infty} \int_{0}^{\infty} \frac{\left(\gamma_{u b}-\gamma_{l b}\right) u_{1} v_{1} \hat{v}_{2}}{8 m_{1} m_{2}} e^{-h^{2}\left(v_{1}^{2}+\hat{v}_{2}^{2}\right)} d v_{1} d \hat{v}_{2}
\end{gathered}
$$

\subsection{Convert Coordinate to Polar Coordinate}

The speed distribution, as shown in the equation above, has a double integral for $v_{1}$ and $\hat{v}_{2}$. By converting Cartesian coordinates to polar coordinates: $v_{1}=r \cos \theta, \hat{v}_{2}=r \sin \theta, d v_{1} d \hat{v}_{2}=r d \theta d r$. We can evaluate the first integral for $\theta$ then evaluate the second integral for $r$ as following.

$$
\begin{aligned}
\mathrm{P}_{\text {new }}^{1}\left(u_{1}\right) & =\frac{2 h^{6}}{m_{1} m_{2} \pi} \int_{u_{1}}^{\infty} \int_{0}^{\pi / 2}\left(\gamma_{u b}-\gamma_{l b}\right) u_{1} r \cos \theta r \sin \theta e^{-h^{2} r^{2}} r d \theta d r \\
& =\frac{h^{6} u_{1}}{m_{1} m_{2} \pi} \int_{u_{1}}^{\infty}\left[\int_{0}^{\pi / 2}\left(\gamma_{u b}-\gamma_{l b}\right) \sin (2 \theta) d \theta\right] e^{-h^{2} r^{2}} r^{3} d r
\end{aligned}
$$

In the flowing section, we will evaluate the integral for $\theta$.

\subsection{Evaluate the Integral for $\theta$}

In this section, we will evaluate the inner integral $\int_{0}^{\pi / 2}\left(\gamma_{u b}-\gamma_{l b}\right) \sin (2 \theta) d \theta$ in Equation $(37)$. Where $\left(\gamma_{u b}-\gamma_{l b}\right)$ could be any of the eight formulas depends on the pre-collision speeds $v_{1}$ and $\hat{v}_{2}$. Because $\left(\gamma_{u b}-\gamma_{l b}\right)$ has a different formula for different $v_{1}$ and $\hat{v}_{2}$, the above integral needs to be separated into different integrals for different regions. For example, the integral path across regions MGF, $\int_{M G F}$, need to be evaluated by three integrals of corresponding formulas $\left(\gamma_{u b}-\gamma_{l b}\right)$ from Table 2 and bounded by the relevant boundary lines from Table 3 as follows.

Where

$$
\begin{gathered}
\int_{M G F} f d \theta=\int_{B L_{7}}^{B L_{2}} f_{M} d \theta+\int_{B L_{2}}^{B L_{4}} f_{G} d \theta+\int_{B L_{4}}^{B L_{1}} f_{F} d \theta \\
=\int_{-\left(\pi / 2-\varphi-\theta_{0}\right)}^{\theta_{0}} f_{M} d \theta+\int_{\theta_{0}}^{\pi / 2+\varphi-\theta_{0}} f_{G} d \theta+\int_{\pi / 2+\varphi-\theta_{0}}^{\pi / 2} f_{F} d \theta
\end{gathered}
$$

$$
f_{R}=\left(\gamma_{u b}-\gamma_{l b}\right)_{R} \sin (2 \theta)
$$

Substituting $\left(\gamma_{u b}-\gamma_{l b}\right)_{R}$ for each region from Table 2 into the above three integrals and evaluating each integral will yield

$$
\int_{M G F} f d \theta=\int_{M G F}\left(\gamma_{u b}-\gamma_{l b}\right) \sin (2 \theta) d \theta=8 m_{1} m_{2} \sin \left(2 \theta_{0}\right)
$$


It can be shown that the integral for $\theta$ integrating through different paths for different $r$ results in the same function of $\theta_{0}$ as

$$
\begin{aligned}
\int_{M P F} f d \theta=\int_{M G F} f d \theta=\int_{M G} f d \theta & =\int_{A M G} f d \theta=\int_{A K G} f d \theta \\
& =\int_{0}^{\pi / 2}\left(\gamma_{u b}-\gamma_{l b}\right) \sin (2 \theta) d \theta=8 m_{1} m_{2} \sin \left(2 \theta_{0}\right)
\end{aligned}
$$

The detailed integrations are shown in Note 2.

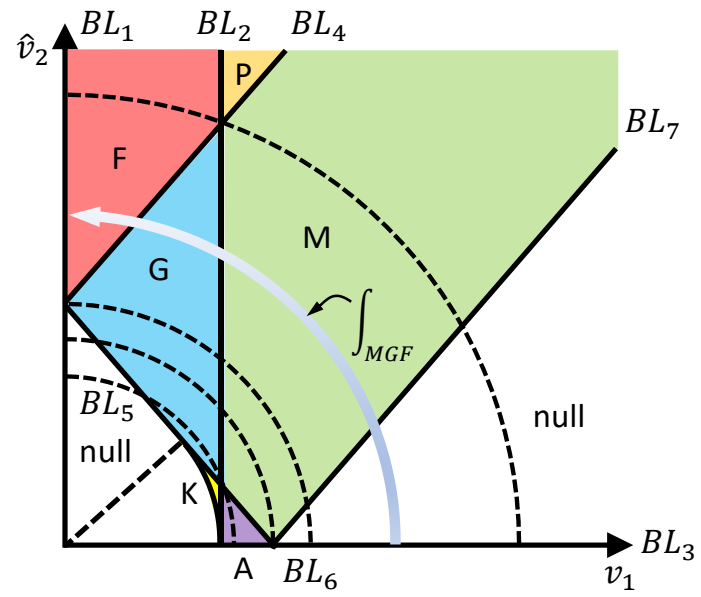

$$
\begin{aligned}
& \int_{M P F}=\int_{B L_{7}}^{B L_{4}} f_{M} d \theta+\int_{B L_{4}}^{B L_{2}} f_{P} d \theta+\int_{B L_{2}}^{B L_{1}} f_{F} d \theta \\
& \int_{M G F}=\int_{B L_{7}}^{B L_{2}} f_{M} d \theta+\int_{B L_{2}}^{B L_{4}} f_{G} d \theta+\int_{B L_{4}}^{B L_{1}} f_{F} d \theta \\
& \int_{M G}=\int_{B L_{7}}^{B L_{2}} f_{M} d \theta+\int_{B L_{2}}^{B L_{5}} f_{G} d \theta \\
& \int_{A M G}=\int_{B L_{3}}^{B L_{6}} f_{A} d \theta+\int_{B L_{6}}^{B L_{2}} f_{M} d \theta+\int_{B L_{2}}^{B L_{5}} f_{G} d \theta \\
& \int_{A K G}=\int_{B L_{3}}^{B L_{2}} f_{A} d \theta+\int_{B L_{2}}^{B L_{6}} f_{K} d \theta+\int_{B L_{6}}^{B L_{5}} f_{G} d \theta \\
& \text { Where } f_{R}=\left(\gamma_{u b}-\gamma_{l b}\right)_{R} \sin (2 \theta) \\
& \text { and }\left(\gamma_{u b}-\gamma_{l b}\right)_{R} \text { for the six regions R } \\
& \text { as (AFGKMP) are listed in Table 2. }
\end{aligned}
$$

Figure 8. Integration paths

\subsection{Evaluate the Integral for $r$}

Substituting Equation (38) into Equation (37) yields

$$
\begin{aligned}
\mathrm{P}_{\text {new }}^{1}\left(u_{1}\right) & =\frac{8 h^{6} u_{1}}{\pi} \int_{u_{1}}^{\infty} \sin \left(2 \theta_{0}\right) e^{-h^{2} r^{2}} r^{3} d r \\
& =\frac{8 h^{6} u_{1}}{\pi} \int_{u_{1}}^{\infty} \frac{2 u_{1}}{r} \sqrt{1-\left(\frac{u_{1}}{r}\right)^{2}} e^{-h^{2} r^{2}} r^{3} d r \\
& =\frac{16 h^{6} u_{1}^{2}}{\pi} \int_{u_{1}}^{\infty} \sqrt{r^{2}-u_{1}^{2}} e^{-h^{2} r^{2}} r d r
\end{aligned}
$$

\subsection{Cange Variable from $r$ to $v$}

Changing the variable from $r$ to $v$ by $v^{2}=r^{2}-u_{1}^{2}, \quad 2 v d v=2 r d r$, and using $\int_{0}^{\infty} \mathrm{P}_{v}(v) d v=$ $\frac{4 h^{3}}{\sqrt{\pi}} \int_{0}^{\infty} v^{2} e^{-h^{2} v^{2}} d v=1$, yields

$$
\begin{aligned}
\mathrm{P}_{\text {new }}^{1}\left(u_{1}\right)= & \frac{16 h^{6} u_{1}^{2}}{\pi} \int_{0}^{\infty} v e^{-h^{2}\left(u_{1}^{2}+v^{2}\right)} v d v \\
= & \frac{4 h^{3}}{\sqrt{\pi}} u_{1}^{2} e^{-h^{2} u_{1}^{2}}\left[\frac{4 h^{3}}{\sqrt{\pi}} \int_{0}^{\infty} v^{2} e^{-h^{2} v^{2}} d v\right] \\
& =\frac{4 h^{3}}{\sqrt{\pi}} u_{1}^{2} e^{-h^{2} u_{1}^{2}}
\end{aligned}
$$

This concludes the derivation of the Maxwell-Boltzmann speed distribution $\mathrm{P}_{\text {new }}^{1}\left(u_{1}\right)$ of the post-collision speed of particle 1 as

$$
\mathrm{P}_{\text {new }}^{1}\left(u_{1}\right)=\frac{4 h_{1}^{3}}{\sqrt{\pi}} u_{1}^{2} e^{-h_{1}^{2} u_{1}^{2}}
$$

The PDF $\mathrm{P}_{n e w}^{2}\left(u_{2}\right)$ of the post-collisiton speed of particle 2 will also be exactly the Maxwell-Boltzmann speed distribution as 


$$
\mathrm{P}_{\text {new }}^{2}\left(u_{2}\right)=\frac{4 h_{2}^{3}}{\sqrt{\pi}} u_{2}^{2} e^{-h_{2}^{2} u_{2}^{2}}
$$

The above PDF $\mathrm{P}_{\text {new }}^{2}\left(u_{2}\right)$ can be concluded by simply treat particle 2 as particle 1 and following the same procedure for getting $\mathrm{P}_{\text {new }}^{1}\left(u_{1}\right)$.

\section{Conclusion and Outlook}

This paper analytically proved the Maxwell-Boltzmann speed distribution and the speed ratio of mixed particles based on particles' collision mechanics. The proof is based on a probability density function of post-collision velocities developed in Sections 3 and 4. The probability density function of the post-collision speed reveals the microscopic mechanics behind the macroscopic phenomenon. It can be used as a mathematical tool in the fields of statistical thermodynamics and kinetic theory.

The derivation of the Maxwell-Boltzmann speed distribution results in another significant outcome: the Maxwell-Boltzmann speed distribution is valid for interactions with extreme mass ratios between molecules and subatomic particles, where the mass ratio is between $10^{-12}$ and $10^{12}$.

This article gives mechanical proof of the Maxwell-Boltzmann speed distribution and the speed ratio for monatomic particles only. The same procedures can be applied to polyatomic particles. The PDF of the post-collision speed must be extended to including the rotation of the molecules unless the rotation is small and its effect can be neglected. Moreover, the procedures may also be applied to charged particles. The chemical characteristic of molecules may be revealed from the speed and spin distributions induced by the mutual interaction of two different molecules. Our method provided in this paper may have a significant impact on this kind of research.

\section{Acknowledgments}

We appreciate the words "Everything is mechanics" said by Chao-Chung Yu (1915 2014), late president of the National Taiwan University. Indeed, the mechanics are the foundation of the universe. We also appreciate Prof. Yu for his encouragement and support.

\section{References}

Avogadro, A. (1822). Essai d'une maniere de determiner les masses relatives des molecules elementaires des corps, et les proportions selon lesquelles elles entrent dans ces combinations. Journal de Physique, 73, 58-76.

Boltzmann, L. (1872). Weitere studien uber das Warmegleichgewicht unter Gasmolekulen, Sitzungsbenchte der Kaiserlichen Akademie der Wissenschaften in Wien. Mathematisch-Naturwissenschaftliche Classe, 66, $275-370$.

Boltzmann, L. (1877). Über die Beziehung zwischen dem zweitenHauptsatz der mechanischen Wärmetheorie und der Wahrscheinlichkeitsrechnung respektive den Sätzen über das Wärmegleichgewicht." Sitzungsberichte der Kaiserlichen Akademie der Wissenschaften in Wien, Mathematisch-Naturwissenschaftliche Classe. Abt. II, 76, 1877, 373-435. Reprinted in Wissenschaftliche Abhandlungen, Vol. II, pp. 164-223, Leipzig: Barth, 1909. https://doi.org/10.1017/CBO9781139381437.011

Brush, S. G. (1966). Kinetic theory Vol.2. Irreversible processes. Pergamon Press, ISBN 9781483155937 1483155935.Selection.2:Ludwig Boltzmann:Further Studies on the Thermal Equilibrium of Gas Molecules (from Sitzungsbenchte der Kaiserlichen Akademie der Wissenschaften,Vienna, 1872), 88-175. https://doi.org/10.1016/B978-0-08-011869-7.50009-6

Garrod, C. (1995). Statistical mechanics and thermodynamics. University of California, Davis, New York Oxford: Oxford University Press, ISBN 0-19-508523-X (IBM software).

Landau L. D., \& Lifshitz, E. M. (1969). Statistical physics. Institute of Physical Problems, U.S.S.R. Academy of Science, Vol.5 of Course of Theoretical Physic, Translated from the Russian by Peierls, E. and Peierls, R.F., London, Paris: Pergamon Press, ISBN 0-201-0-04167-7.

Lin, T.-W., \& Lin, H. (2019). Mechanical Proof of the Maxwell Speed Distribution, International Journal of Statistics and Probability, 8(2), 90-98. https://doi.org/10.5539/ijsp.v8n2p90

Maudlin, T. (2013). How to derive the equilibrium velocity distribution two ways (Neither of which is what you are expecting). Cosmology Summer School, Santa Cruz, July 26, 2013, NYU. Retrieved from http://hipacc.ucsc.edu/IPC2013/slides/130626_TimMaudlin.pdf

Maxwell, J. C. (1860a). V. Illustrations of the dynamical theory of gases, Part I. On the motions and collisions of perfectly elastic spheres, The London, Edinburgh, and Dubin Philosophical Magazine and Journal of Science, $4^{\text {th }}$ Series, 19, 19-32. https://doi.org/10.1080/14786446808639951 
Maxwell, J. C. (1860b). II. Illustrations of the dynamical theory of gases, Part II. On the process of diffusion of two or more kinds of moving particles among one another, The London. Edinburgh, and Dubin Philosophical Magazine and Journal of Science, $4^{\text {th }}$ Series, 20, 21-37, https://doi.org/10.1080/14786446008642902

Maxwell, J. C. (1867). XV. On the dynamical theory of gases, The London, Edinburgh, and Dubin Philosophical Magazine and Journal of Science, $4^{\text {th }}$ Series, 35, 129-145, \& 36, 185-217, https://doi.org/10.1080/14786446808639951, https://doi.org/10.1080/14786446808639963

McQuarrie, D. A. (1976). Statistical mechanics. Indiana University, Harper Collins Publishers, ISBN 06-044366-9.

\section{Notes}

\section{Note 1.}

To solve for $\cos \alpha_{l b, u b}$ from the following equation.

$$
u_{1}=\left|m_{1} \sqrt{v_{1}^{2}+\left(\frac{m_{2}}{m_{1}} v_{2}\right)^{2}+2 v_{1}\left(\frac{m_{2}}{m_{1}} v_{2}\right) \cos \alpha_{l b, u b}} \pm m_{2} \sqrt{v_{1}^{2}+v_{2}^{2}-2 v_{1} v_{2} \cos \alpha_{l b, u b}}\right|
$$

Let $c_{l b, u b} \equiv \cos \alpha_{l b, u b}$, and square to get

$$
\begin{aligned}
u_{1}^{2} & =\left(m_{1}^{2} v_{1}^{2}+m_{2}^{2} v_{2}^{2}+2 m_{1} m_{2} v_{1} v_{2} c_{l b, u b}\right)+\left(m_{2}^{2} v_{1}^{2}+m_{2}^{2} v_{2}^{2}-2 m_{2}^{2} v_{1} v_{2} c_{l b, u b}\right) \\
& \pm 2 \sqrt{\left(m_{1}^{2} v_{1}^{2}+m_{2}^{2} v_{2}^{2}+2 m_{1} m_{2} v_{1} v_{2} c_{l b, u b}\right)\left(m_{2}^{2} v_{1}^{2}+m_{2}^{2} v_{2}^{2}-2 m_{2}^{2} v_{1} v_{2} c_{l b, u b}\right)}
\end{aligned}
$$

Rearrange and square again to get

$$
\begin{gathered}
{\left[\left(m_{1}^{2} v_{1}^{2}+m_{2}^{2} v_{2}^{2}+2 m_{1} m_{2} v_{1} v_{2} c_{l b, u b}\right)+\left(m_{2}^{2} v_{1}^{2}+m_{2}^{2} v_{2}^{2}-2 m_{2}^{2} v_{1} v_{2} c_{l b, u b}\right)-u_{1}^{2}\right]^{2}} \\
-4\left(m_{1}^{2} v_{1}^{2}+m_{2}^{2} v_{2}^{2}+2 m_{1} m_{2} v_{1} v_{2} c_{l b, u b}\right)\left(m_{2}^{2} v_{1}^{2}+m_{2}^{2} v_{2}^{2}-2 m_{2}^{2} v_{1} v_{2} c_{l b, u b}\right)=0
\end{gathered}
$$

or

$$
\begin{aligned}
& \left(m_{1}^{2} v_{1}^{2}+m_{2}^{2} v_{2}^{2}+2 m_{1} m_{2} v_{1} v_{2} c_{l b, u b}\right)^{2}+\left(m_{2}^{2} v_{1}^{2}+m_{2}^{2} v_{2}^{2}-2 m_{2}^{2} v_{1} v_{2} c_{l b, u b}\right)^{2}+u_{1}^{4} \\
& -2 u_{1}^{2}\left(m_{1}^{2} v_{1}^{2}+m_{2}^{2} v_{2}^{2}+2 m_{1} m_{2} v_{1} v_{2} c_{l b, u b}+m_{2}^{2} v_{1}^{2}+m_{2}^{2} v_{2}^{2}-2 m_{2}^{2} v_{1} v_{2} c_{l b, u b}\right) \\
& -2\left(m_{1}^{2} v_{1}^{2}+m_{2}^{2} v_{2}^{2}+2 m_{1} m_{2} v_{1} v_{2} c_{l b, u b}\right)\left(m_{2}^{2} v_{1}^{2}+m_{2}^{2} v_{2}^{2}-2 m_{2}^{2} v_{1} v_{2} c_{l b, u b}\right)=0
\end{aligned}
$$

or

$$
A^{\prime} c_{l b, u b}^{2}+2 B^{\prime} c_{l b, u b}+C^{\prime}=0
$$

Where

$$
\begin{aligned}
A^{\prime}= & \left(2 m_{1} m_{2} v_{1} v_{2}\right)^{2}+\left(2 m_{2}^{2} v_{1} v_{2}\right)^{2}+2\left(2 m_{1} m_{2} v_{1} v_{2}\right)\left(2 m_{2}^{2} v_{1} v_{2}\right) \\
= & 4\left(m_{1} m_{2}+m_{2}^{2}\right)^{2} v_{1}^{2} v_{2}^{2}=4 m_{2}^{2} v_{1}^{2} v_{2}^{2}=4 m_{1} m_{2} v_{1}^{2} \hat{v}_{2}^{2} \\
C^{\prime}= & u_{1}^{4}-2 u_{1}^{2}\left(m_{1}^{2} v_{1}^{2}+m_{2}^{2} v_{2}^{2}+m_{2}^{2} v_{1}^{2}+m_{2}^{2} v_{2}^{2}\right) \\
& +\left(m_{1}^{2} v_{1}^{2}+m_{2}^{2} v_{2}^{2}\right)^{2}+\left(m_{2}^{2} v_{1}^{2}+m_{2}^{2} v_{2}^{2}\right)^{2}-2\left(m_{1}^{2} v_{1}^{2}+m_{2}^{2} v_{2}^{2}\right)\left(m_{2}^{2} v_{1}^{2}+m_{2}^{2} v_{2}^{2}\right) \\
= & u_{1}^{4}-2 u_{1}^{2}\left(\left(m_{1}^{2}+m_{2}^{2}\right) v_{1}^{2}+2 m_{2}^{2} v_{2}^{2}\right)+\left(m_{1}^{2}-m_{2}^{2}\right)^{2} v_{1}^{4} \\
= & u_{1}^{4}-2 u_{1}^{2}\left(\left(m_{1}^{2}+m_{2}^{2}\right) v_{1}^{2}+2 m_{1} m_{2} \hat{v}_{2}^{2}\right)+\left(m_{1}-m_{2}\right)^{2} v_{1}^{4} \\
B^{\prime}= & 2 m_{1} m_{2} v_{1} v_{2}\left(m_{1}^{2} v_{1}^{2}+m_{2}^{2} v_{2}^{2}\right)-2 m_{2}^{2} v_{1} v_{2}\left(m_{2}^{2} v_{1}^{2}+m_{2}^{2} v_{2}^{2}\right) \\
& +2 m_{2}^{2} v_{1} v_{2}\left(m_{1}^{2} v_{1}^{2}+m_{2}^{2} v_{2}^{2}\right)-2 m_{1} m_{2} v_{1} v_{2}\left(m_{2}^{2} v_{1}^{2}+m_{2}^{2} v_{2}^{2}\right) \\
& +2 u_{1}^{2}\left(m_{2}^{2}-m_{1} m_{2}\right) v_{1} v_{2} \\
= & 2 m_{1} m_{2} v_{1} v_{2}\left(m_{1}^{2} v_{1}^{2}-m_{2}^{2} v_{1}^{2}\right)+2 m_{2}^{2} v_{1} v_{2}\left(m_{1}^{2} v_{1}^{2}-m_{2}^{2} v_{1}^{2}\right)
\end{aligned}
$$




$$
\begin{aligned}
& +2 u_{1}^{2}\left(m_{2}^{2}-m_{1} m_{2}\right) v_{1} v_{2} \\
= & 2 m_{2} v_{1} v_{2}\left(m_{1}^{2}-m_{2}^{2}\right) v_{1}^{2}+2 u_{1}^{2}\left(m_{2}^{2}-m_{1} m_{2}\right) v_{1} v_{2} \\
= & 2 m_{2} v_{1} v_{2}\left(m_{1}-m_{2}\right)\left(v_{1}^{2}-u_{1}^{2}\right) \\
= & \sqrt{4 m_{1} m_{2}} v_{1} \hat{v}_{2}\left(m_{1}-m_{2}\right)\left(v_{1}^{2}-u_{1}^{2}\right) \\
B^{\prime 2}-A^{\prime} C^{\prime}= & 4 m_{1} m_{2} v_{1}^{2} \hat{v}_{2}^{2} \\
& \quad\left(\left(m_{1}-m_{2}\right)^{2}\left(v_{1}^{2}-u_{1}^{2}\right)^{2}-u_{1}^{4}+2 u_{1}^{2}\left(\left(m_{1}^{2}+m_{2}^{2}\right) v_{1}^{2}+2 m_{1} m_{2} \hat{v}_{2}^{2}\right)-\left(m_{1}-m_{2}\right)^{2} v_{1}^{4}\right) \\
= & 4 m_{1} m_{2} v_{1}^{2} \hat{v}_{2}^{2} u_{1}^{2}\left(4 m_{1} m_{2}\left(v_{1}^{2}+\hat{v}_{2}^{2}\right)-u_{1}^{2}\left(1-\left(m_{1}-m_{2}\right)^{2}\right)\right) \\
= & \left(4 m_{1} m_{2} v_{1} \hat{v}_{2} u_{1}\right)^{2}\left(v_{1}^{2}+\hat{v}_{2}^{2}-u_{1}^{2}\right)
\end{aligned}
$$

Therefore, the $c_{l b, u b}$ in Equation (N1) are given by

$$
\begin{gathered}
c_{l b, u b}=\frac{-\sqrt{4 m_{1} m_{2}} v_{1} \hat{v}_{2}\left(m_{1}-m_{2}\right)\left(v_{1}^{2}-u_{1}^{2}\right) \pm 4 m_{1} m_{2} v_{1} \hat{v}_{2} u_{1} \sqrt{v_{1}^{2}+\hat{v}_{2}^{2}-u_{1}^{2}}}{4 m_{1} m_{2} v_{1}^{2} \hat{v}_{2}^{2}} \\
=\frac{\left(m_{2}-m_{1}\right)\left(v_{1}^{2}-u_{1}^{2}\right) \pm \sqrt{4 m_{1} m_{2}} u_{1} \sqrt{v_{1}^{2}+\hat{v}_{2}^{2}-u_{1}^{2}}}{\sqrt{4 m_{1} m_{2}} v_{1} \hat{v}_{2}}
\end{gathered}
$$

By using $r=\sqrt{v_{1}^{2}+\hat{v}_{2}^{2}}, \theta=\tan ^{-1}\left(\frac{\hat{v}_{2}}{v_{1}}\right), \theta_{o}=\cos ^{-1}\left(\frac{u_{1}}{r}\right), \varphi=\tan ^{-1}\left(\frac{m_{2}-m_{1}}{\sqrt{4 m_{1} m_{2}}}\right)$,

the equation above becomes

$$
\begin{gathered}
c_{l b, u b}=\frac{\sin \varphi\left(\cos ^{2} \theta-\cos ^{2} \theta_{o}\right) \pm \cos \varphi \cos \theta_{o} \sin \theta_{o}}{\cos \varphi \cos \theta \sin \theta}=\frac{\sin \varphi\left(\cos (2 \theta)-\cos \left(2 \theta_{o}\right)\right) \pm \cos \varphi \sin \left(2 \theta_{o}\right)}{\cos \varphi \sin (2 \theta)} \\
=\frac{\sin \varphi \cos (2 \theta) \pm \sin \left(2 \theta_{o} \mp \varphi\right)}{\cos \varphi \sin (2 \theta)}
\end{gathered}
$$

Because the value of any cosine function cannot be smaller than -1 or larger than +1 , we must limit the values of $\cos \alpha_{l b, u b}$ between -1 and +1 as

$$
\begin{gathered}
\cos \alpha_{l b}=\min \left(c_{l b},+1\right)=\min \left(\frac{\sin \varphi \cos (2 \theta)+\sin \left(2 \theta_{o}-\varphi\right)}{\cos \varphi \sin (2 \theta)},+1\right) \\
\cos \alpha_{u b}=\max \left(c_{u b},-1\right)=\max \left(\frac{\sin \varphi \cos (2 \theta)-\sin \left(2 \theta_{o}+\varphi\right)}{\cos \varphi \sin (2 \theta)},-1\right)
\end{gathered}
$$

Note 2.

a) Integration paths across the regions (AKGF) and ( $\left.\mathrm{AKG}^{\prime}\right)$ :

A: $\int_{B L_{3}}^{B L_{2}} f_{A} d \theta=\int_{0}^{\theta_{0}}(4 \theta) \sin (2 \theta) d \theta=[-2 \theta \cos (2 \theta)]_{0}^{\theta_{0}}+[\sin (2 \theta)]_{0}^{\theta_{0}}$

$=-2 \theta_{0} \cos \left(2 \theta_{0}\right)+\sin \left(2 \theta_{0}\right)$

$\mathrm{K}: \int_{B L_{2}}^{B L_{6}} f_{K} d \theta=\int_{\theta_{0}}^{\frac{\pi}{2}-\varphi-\theta_{0}}\left(4 \theta_{0}\right) \sin (2 \theta) d \theta=\left[-2 \theta_{0} \cos (2 \theta)\right]_{\theta_{0}}^{\frac{\pi}{2}-\varphi-\theta_{0}}$

$=-2 \theta_{0} \cos \left(\pi-2 \varphi-2 \theta_{0}\right)+2 \theta_{0} \cos \left(2 \theta_{0}\right)$ 
$\mathrm{G}^{\prime}: \int_{B L_{6}}^{B L_{5}} f_{G} d \theta=\int_{\frac{\pi}{2}-\varphi-\theta_{0}}^{\frac{\pi}{2}-\varphi+\theta_{0}}\left(\pi-2 \varphi-2 \theta+2 \theta_{0}\right) \sin (2 \theta) d \theta$

$$
\begin{aligned}
& =\frac{1}{2}\left[-\left(\pi-2 \varphi-2 \theta+2 \theta_{0}\right) \cos (2 \theta)\right]_{\frac{\pi}{2}-\varphi-\theta_{0}}^{\frac{\pi}{2}-\varphi+\theta_{0}}-\frac{1}{2}[\sin (2 \theta)]_{\frac{\pi}{2}-\varphi-\theta_{0}}^{\frac{\pi}{2}-\varphi+\theta_{0}} \\
& =2 \theta_{0} \cos \left(\pi-2 \varphi-2 \theta_{0}\right)+\frac{1}{2} \sin \left(\pi+2 \varphi-2 \theta_{0}\right)+\frac{1}{2} \sin \left(\pi-2 \varphi-2 \theta_{0}\right)
\end{aligned}
$$

$\mathrm{G}: \int_{B L_{6}}^{B L_{4}} f_{G} d \theta=\int_{\frac{\pi}{2}-\varphi-\theta_{0}}^{\frac{\pi}{2}+\varphi-\theta_{0}}\left(\pi-2 \varphi-2 \theta+2 \theta_{0}\right) \sin (2 \theta) d \theta$

$$
\begin{gathered}
=\frac{1}{2}\left[-\left(\pi-2 \varphi-2 \theta+2 \theta_{0}\right) \cos (2 \theta)\right]_{\frac{\pi}{2}-\varphi-\theta_{0}}^{\frac{\pi}{2}+\varphi-\theta_{0}}-\frac{1}{2}[\sin (2 \theta)]_{\frac{\pi}{2}-\varphi-\theta_{0}}^{\frac{\pi}{2}+\varphi-\theta_{0}} \\
=\left(2 \varphi-2 \theta_{0}\right) \cos \left(\pi+2 \varphi-2 \theta_{0}\right)+2 \theta_{0} \cos \left(\pi-2 \varphi-2 \theta_{0}\right) \\
\quad-\frac{1}{2} \sin \left(\pi+2 \varphi-2 \theta_{0}\right)+\frac{1}{2} \sin \left(\pi-2 \varphi-2 \theta_{0}\right)
\end{gathered}
$$

$\mathrm{F}: \int_{B L_{4}}^{B L_{1}} f_{F} d \theta=\int_{\frac{\pi}{2}+\varphi-\theta_{0}}^{\frac{\pi}{2}}(2 \pi-4 \theta) \sin (2 \theta) d \theta$

$$
\begin{aligned}
& =[-(\pi-2 \theta) \cos (2 \theta)]_{\frac{\pi}{2}+\varphi-\theta_{0}}^{\frac{\pi}{2}}-[\sin (2 \theta)]_{\frac{\pi}{2}+\varphi-\theta_{0}}^{\frac{\pi}{2}} \\
& =-\left(2 \varphi-2 \theta_{0}\right) \cos \left(\pi+2 \varphi-2 \theta_{0}\right)+\sin \left(\pi+2 \varphi-2 \theta_{0}\right)
\end{aligned}
$$

Combine the results to get

$$
\begin{aligned}
& \int_{A K G F}\left(\gamma_{2}-\gamma_{1}\right) \sin (2 \theta) d \theta=\int_{A K G^{\prime}}\left(\gamma_{2}-\gamma_{1}\right) \sin (2 \theta) d \theta \\
& =\sin \left(2 \theta_{0}\right)+\frac{1}{2} \sin \left(\pi-2 \varphi-2 \theta_{0}\right)+\frac{1}{2} \sin \left(\pi+2 \varphi-2 \theta_{0}\right) \\
& =\sin \left(2 \theta_{0}\right)+\sin \left(\pi-2 \theta_{0}\right) \cos (2 \varphi)=(1+\cos (2 \varphi)) \sin \left(2 \theta_{0}\right) \\
& =2 \cos ^{2} \varphi \sin \left(2 \theta_{0}\right)=8 m_{1} m_{2} \sin \left(2 \theta_{0}\right)
\end{aligned}
$$

It is interesting to note that all the terms with a cosine function are canceled.

For $m_{1}=m_{2}=1 / 2, \varphi=0$, only two integration paths are needed: (AKF) and (APF).

$$
\begin{aligned}
& \int_{A K F}\left(\gamma_{2}-\gamma_{1}\right) \sin (2 \theta) d \theta=\int_{A P K}\left(\gamma_{2}-\gamma_{1}\right) \sin (2 \theta) d \theta \\
& \quad=\sin \left(2 \theta_{0}\right)+\sin \left(\pi-2 \theta_{0}\right)=2 \sin \left(2 \theta_{0}\right)=8 m_{1} m_{2} \sin \left(2 \theta_{0}\right)
\end{aligned}
$$

b) Integration paths across the regions (AMGF), (AMG'), and (M'G'):

$$
\begin{aligned}
\mathrm{A}: & \int_{B L_{3}}^{B L_{6}} f_{A} d \theta=\int_{0}^{\frac{\pi}{2}-\varphi-\theta_{0}}(4 \theta) \sin (2 \theta) d \theta=[-2 \theta \cos (2 \theta)]_{0}^{\frac{\pi}{2}-\varphi-\theta_{0}}+[\sin (2 \theta)]_{0}^{\frac{\pi}{2}-\varphi-\theta_{0}} \\
& =-\left(\pi-2 \varphi-2 \theta_{0}\right) \cos \left(\pi-2 \varphi-2 \theta_{0}\right)+\sin \left(\pi-2 \varphi-2 \theta_{0}\right)
\end{aligned}
$$


$\mathrm{M}: \int_{B L_{6}}^{B L_{2}} f_{M} d \theta=\int_{\frac{\pi}{2}-\varphi-\theta_{0}}^{\theta_{0}}\left(\pi-2 \varphi+2 \theta-2 \theta_{0}\right) \sin (2 \theta) d \theta$

$$
\begin{gathered}
=\frac{1}{2}\left[-\left(\pi-2 \varphi+2 \theta-2 \theta_{0}\right) \cos (2 \theta)\right]_{\frac{\pi}{2}-\varphi-\theta_{0}}^{\theta_{0}}+\frac{1}{2}[\sin (2 \theta)]_{\frac{\pi}{2}-\varphi-\theta_{0}}^{\theta_{0}} \\
=\left(\pi-2 \varphi-2 \theta_{0}\right) \cos \left(\pi-2 \varphi-2 \theta_{0}\right)-\frac{1}{2}(\pi-2 \varphi) \cos \left(2 \theta_{0}\right) \\
-\frac{1}{2} \sin \left(\pi-2 \varphi-2 \theta_{0}\right)+\frac{1}{2} \sin \left(2 \theta_{0}\right)
\end{gathered}
$$

$\mathrm{M}^{\prime}: \int_{B L_{7}}^{B L_{2}} f_{M} d \theta=\int_{-\left(\frac{\pi}{2}-\varphi-\theta_{0}\right)}^{\theta_{0}}\left(\pi-2 \varphi+2 \theta-2 \theta_{0}\right) \sin (2 \theta) d \theta$

$$
\begin{aligned}
& =\frac{1}{2}\left[-\left(\pi-2 \varphi+2 \theta-2 \theta_{0}\right) \cos (2 \theta)\right]_{-\left(\frac{\pi}{2}-\varphi-\theta_{0}\right)}^{\theta_{0}}+\frac{1}{2}[\sin (2 \theta)]_{-\left(\frac{\pi}{2}-\varphi-\theta_{0}\right)}^{\theta_{0}} \\
& =-\frac{1}{2}(\pi-2 \varphi) \cos \left(2 \theta_{0}\right)+\frac{1}{2} \sin \left(\pi-2 \varphi-2 \theta_{0}\right)+\frac{1}{2} \sin \left(2 \theta_{0}\right)
\end{aligned}
$$

$\mathrm{G}^{\prime}: \int_{B L_{2}}^{B L_{5}} f_{G} d \theta=\int_{\theta_{0}}^{\frac{\pi}{2}-\varphi+\theta_{0}}\left(\pi-2 \varphi-2 \theta+2 \theta_{0}\right) \sin (2 \theta) d \theta$

$$
\begin{aligned}
& =\frac{1}{2}\left[-\left(\pi-2 \varphi-2 \theta+2 \theta_{0}\right) \cos (2 \theta)\right]_{\theta_{0}}^{\frac{\pi}{2}-\varphi+\theta_{0}}-\frac{1}{2}[\sin (2 \theta)]_{\theta_{0}}^{\frac{\pi}{2}-\varphi+\theta_{0}} \\
& =\frac{1}{2}(\pi-2 \varphi) \cos \left(2 \theta_{0}\right)+\frac{1}{2} \sin \left(\pi+2 \varphi-2 \theta_{0}\right)+\frac{1}{2} \sin \left(2 \theta_{0}\right)
\end{aligned}
$$

$\mathrm{G}: \int_{B L_{2}}^{B L_{4}} f_{G} d \theta=\int_{\theta_{0}}^{\frac{\pi}{2}+\varphi-\theta_{0}}\left(\pi-2 \varphi-2 \theta+2 \theta_{0}\right) \sin (2 \theta) d \theta$

$$
\begin{gathered}
=\frac{1}{2}\left[-\left(\pi-2 \varphi-2 \theta+2 \theta_{0}\right) \cos (2 \theta)\right]_{\theta_{0}}^{\frac{\pi}{2}+\varphi-\theta_{0}}-\frac{1}{2}[\sin (2 \theta)]_{\theta_{0}}^{\frac{\pi}{2}+\varphi-\theta_{0}} \\
=\left(2 \varphi-2 \theta_{0}\right) \cos \left(\pi+2 \varphi-2 \theta_{0}\right)+\frac{1}{2}(\pi-2 \varphi) \cos \left(2 \theta_{0}\right) \\
-\frac{1}{2} \sin \left(\pi+2 \varphi-2 \theta_{0}\right)+\frac{1}{2} \sin \left(2 \theta_{0}\right)
\end{gathered}
$$

$\mathrm{F}: \int_{B L_{4}}^{B L_{1}} f_{F} d \theta=\int_{\frac{\pi}{2}+\varphi-\theta_{0}}^{\frac{\pi}{2}}(2 \pi-4 \theta) \sin (2 \theta) d \theta$

$$
=-\left(2 \varphi-2 \theta_{0}\right) \cos \left(\pi+2 \varphi-2 \theta_{0}\right)+\sin \left(\pi+2 \varphi-2 \theta_{0}\right)
$$

Combine the results to get

$$
\begin{aligned}
& \int_{A M G F}\left(\gamma_{2}-\gamma_{1}\right) \sin (2 \theta) d \theta=\int_{A M G^{\prime}}\left(\gamma_{2}-\gamma_{1}\right) \sin (2 \theta) d \theta=\int_{M^{\prime} G^{\prime}}\left(\gamma_{2}-\gamma_{1}\right) \sin (2 \theta) d \theta \\
& \quad=\frac{1}{2} \sin \left(\pi-2 \varphi-2 \theta_{0}\right)+\frac{1}{2} \sin \left(2 \theta_{0}\right)+\frac{1}{2} \sin \left(\pi+2 \varphi-2 \theta_{0}\right)+\frac{1}{2} \sin \left(2 \theta_{0}\right) \\
& =\sin \left(2 \theta_{0}\right)+\sin \left(\pi-2 \theta_{0}\right) \cos (2 \varphi)=8 m_{1} m_{2} \sin \left(2 \theta_{0}\right)
\end{aligned}
$$


The integration path (M'G') is the only path for very small $m_{1}$.

c) Integration paths across the regions (AMPF) and (M'PF):

$\mathrm{A}: \int_{B L_{3}}^{B L_{6}} f_{A} d \theta=\int_{0}^{\frac{\pi}{2}-\varphi-\theta_{0}}(4 \theta) \sin (2 \theta) d \theta$

$=-\left(\pi-2 \varphi-2 \theta_{0}\right) \cos \left(\pi-2 \varphi-2 \theta_{0}\right)+\sin \left(\pi-2 \varphi-2 \theta_{0}\right)$

$\mathrm{M}: \int_{B L_{6}}^{B L_{4}} f_{M} d \theta=\int_{\frac{\pi}{2}-\varphi-\theta_{0}}^{\frac{\pi}{2}+\varphi-\theta_{0}}\left(\pi-2 \varphi+2 \theta-2 \theta_{0}\right) \sin (2 \theta) d \theta$

$$
\begin{gathered}
=\frac{1}{2}\left[-\left(\pi-2 \varphi+2 \theta-2 \theta_{0}\right) \cos (2 \theta)\right]_{\frac{\pi}{2}-\varphi-\theta_{0}}^{\frac{\pi}{2}+\varphi-\theta_{0}}+\frac{1}{2}[\sin (2 \theta)]_{\frac{\pi}{2}-\varphi-\theta_{0}}^{\frac{\pi}{2}+\varphi-\theta_{0}} \\
=-\left(\pi-2 \theta_{0}\right) \cos \left(\pi+2 \varphi-2 \theta_{0}\right)+\left(\pi-2 \varphi-2 \theta_{0}\right) \cos \left(\pi-2 \varphi-2 \theta_{0}\right) \\
\quad+\frac{1}{2} \sin \left(\pi+2 \varphi-2 \theta_{0}\right)-\frac{1}{2} \sin \left(\pi-2 \varphi-2 \theta_{0}\right)
\end{gathered}
$$

$\mathrm{M}^{\prime}: \int_{B L_{7}}^{B L_{4}} f_{M} d \theta=\int_{-\left(\frac{\pi}{2}-\varphi-\theta_{0}\right)}^{\frac{\pi}{2}+\varphi-\theta_{0}}\left(\pi-2 \varphi+2 \theta-2 \theta_{0}\right) \sin (2 \theta) d \theta$

$$
\begin{aligned}
& =\frac{1}{2}\left[-\left(\pi-2 \varphi+2 \theta-2 \theta_{0}\right) \cos (2 \theta)\right]_{-\left(\frac{\pi}{2}-\varphi-\theta_{0}\right)}^{\frac{\pi}{2}+\varphi-\theta_{0}}+\frac{1}{2}[\sin (2 \theta)]_{-\left(\frac{\pi}{2}-\varphi-\theta_{0}\right)}^{\frac{\pi}{2}+\varphi-\theta_{0}} \\
& =-\left(\pi-2 \theta_{0}\right) \cos \left(\pi+2 \varphi-2 \theta_{0}\right)+\frac{1}{2} \sin \left(\pi+2 \varphi-2 \theta_{0}\right)+\frac{1}{2} \sin \left(\pi-2 \varphi-2 \theta_{0}\right)
\end{aligned}
$$

$\mathrm{P}: \int_{B L_{4}}^{B L_{2}} f_{P} d \theta=\int_{\frac{\pi}{2}+\varphi-\theta_{0}}^{\theta_{0}}\left(2 \pi-4 \theta_{0}\right) \sin (2 \theta) d \theta=\left[-\left(\pi-2 \theta_{0}\right) \cos (2 \theta)\right]_{\frac{\pi}{2}+\varphi-\theta_{0}}^{\theta_{0}}$ $=\left(\pi-2 \theta_{0}\right) \cos \left(\pi+2 \varphi-2 \theta_{0}\right)-\left(\pi-2 \theta_{0}\right) \cos \left(2 \theta_{0}\right)$

$\mathrm{F}: \int_{B L_{2}}^{B L_{1}} f_{F} d \theta=\int_{\theta_{0}}^{\frac{\pi}{2}}(2 \pi-4 \theta) \sin (2 \theta) d \theta=[-(\pi-2 \theta) \cos (2 \theta)]_{\theta_{0}}^{\frac{\pi}{2}}-[\sin (2 \theta)]_{\theta_{0}}^{\frac{\pi}{2}}$ $=\left(\pi-2 \theta_{0}\right) \cos \left(2 \theta_{0}\right)+\sin \left(2 \theta_{0}\right)$

Combine the results to get

$$
\begin{aligned}
& \int_{A M P F}\left(\gamma_{2}-\gamma_{1}\right) \sin (2 \theta) d \theta=\int_{M I P F}\left(\gamma_{2}-\gamma_{1}\right) \sin (2 \theta) d \theta \\
& =\frac{1}{2} \sin \left(\pi-2 \varphi-2 \theta_{0}\right)+\frac{1}{2} \sin \left(\pi+2 \varphi-2 \theta_{0}\right)+\sin \left(2 \theta_{0}\right) \\
& =\sin \left(\pi-2 \theta_{0}\right) \cos (2 \varphi)+\sin \left(2 \theta_{0}\right)=8 m_{1} m_{2} \sin \left(2 \theta_{0}\right)
\end{aligned}
$$

\section{Copyrights}

Copyright for this article is retained by the author(s), with first publication rights granted to the journal.

This is an open-access article distributed under the terms and conditions of the Creative Commons Attribution license (http://creativecommons.org/licenses/by/4.0/). 